\title{
2-D preplanetary accretion disks
}

\section{Hydrodynamics, chemistry, and mixing processes}

\author{
W. M. Tscharnuter and H.-P. Gail
}

\begin{abstract}
Zentrum für Astronomie (ZAH), Institut für Theoretische Astrophysik (ITA), University of Heidelberg, Albert-Ueberle-Str. 2 , 69120 Heidelberg, Germany

e-mail: wmt@ita.uni-heidelberg.de
\end{abstract}

Received 10 June 2006 / Accepted 6 December 2006

\begin{abstract}
Aims. We outline a numerical method to calculate spatially two-dimensional (2-D) reactive flows and mixing processes in preplanetary accretion disks and present first results. The numerical efficiency and robustness is demonstrated by following the hydrodynamical and chemical evolution of the disk from a highly non-stationary dynamical "switch-on" phase asymptotically into the quasi-stationary, viscous accretion regime. One major question we address is the $\mathrm{C}-, \mathrm{H}-, \mathrm{O}$-chemistry. The leit-motif of our investigation is the attempt to preserve as much consistency as possible when modelling the hydrodynamical, chemical, transport/mixing processes and their mutual interactions in preplanetary disks.

Methods. We use an explicit scheme for solving the Navier-Stokes equations combined with an implicit solver for the energy equation. The viscosity coefficient is modelled according to the so-called $\beta$-prescription of "turbulent" viscosity. In contrast to the well-known $\alpha$-viscosity, the $\beta$-parameterization of the viscosity warrants physical consistency if self-gravitation of the disk material is to be taken into account. However, up to now we have neglected self-gravitation. For the radiative energy transport we have adopted the (grey) Eddington approximation. The opacity is assumed to be caused by microscopic dust particles. Diffusive mixing of the various chemical species is modelled by taking the diffusion coefficient, $D$, proportional to the (turbulent) viscosity, $v_{\text {turb }}$. For comparison purposes, we have considered two extreme choices of the Schmidt number, $\mathcal{S}:=v_{\text {turb }} / D$, that is, $\mathcal{S}=1\left(D=v_{\text {turb }}\right)$ and $\mathcal{S}=\infty(D=0$, i.e., no diffusive mixing at all), respectively. We have not yet included coagulation processes and grain growth.

Results. The main outcome of the 2-D simulations so far carried out is a characteristic circulation pattern of the quasi-stationary accretion flow: Near the disk's equatorial plane which is assumed to be a plane of symmetry the material moves in the outward direction, whereas the accretion flow proper develops in higher altitudes of the disk. Species that are produced or undergo chemical reactions in the warm inner zones of the disk are advectively transported into the cool outer regions. At the same time, they either diffusively mix up with the surrounding material or freeze out on the dust grains to form "ice"-coated particles. By virtue of the large-scale circulation, which is driven by viscous angular momentum transfer, advective transport dominates diffusive mixing in the outer part of the disk.
\end{abstract}

Key words. accretion, accretion disks - hydrodynamics - diffusion - astrochemistry - solar system: formation

\section{Introduction}

Existing disk models are based on several approximations, in particular, if emphasis is laid on the investigation of the complex chemical and mineralogical processes in protoplanetary disks. One salient feature of accretion disks is the fact that they are geometrically thin ("pancake"-like) objects. Hence, the simplest way to construct disk models is to use quantities that are, together with the structure equations, properly averaged over the vertical $(z-)$ direction parallel to the axis of rotation in a cylindrical system of polar coordinates $(r, \phi, z)$.

The simplest non-trivial disk models are the spatially 1-D, one-zone models. Only the radial coordinate, $r$, is retained, the $z$-dependence is completely eliminated and all relations referring to the vertical direction are omitted. Since the viscous timescale is, in general, orders of magnitude larger than the dynamical ("Keplerian") revolution time, the Keplerian rotation law can be assumed to be established at every instant of time. This statement remains true as long as the self-gravitation of the disk material can be neglected. The slow radial drift is completely governed by the efficiency of turbulent friction in redistributing angular momentum. So, in the 1-D approach with a given viscosity parameter, only the detailed conservation of mass and angular momentum has to be observed.

Nevertheless, assured a thermally steady state, it is even possible to define a radial distribution of an "effective" temperature as an equivalent measure of the radiative flux by demanding the flux emitted from the disk's surface to be equal to the locally dissipated energy within the disk. This temperature definition in the 1-D approximation relies entirely on an energy balance argument, no detailed radiative transfer needs to be considered. Incidentally, here and in the following, too, it is tacitely assumed that energy transport takes place exclusively in the vertical direction where the gradients are much steeper than in the radial direction.

Within the more general framework of the so-called $(1+1)$-approximation the disk is assumed to be vertically in strict hydrostatic equilibrium. The basic evolutionary equations are, as in the pure 1-D case, the $z$-averaged equations expressing mass and angular momentum conservation. The method of determining the hydrostatic vertical stratification of the density, temperature, and pressure at each radial distance is very much the same as the one used for stellar structure calculations. Of course, to 
be able to proceed in this manner, gradients in the radial direction are neglected. The connection between the basic 1-D models and the $z$-hydrostatic assumption is the obvious demand on the $z$-integrated density, i.e., the column density, to be always equal to the surface density which is known by solving the basic 1-D evolutionary equations.

Preplanetary disk models based on the one-zone approximation have been published by Ruden \& Pollack (1991); Cassen (1994); Schmitt et al. (1997); Gail (1998); more elaborate (1+1)-D model calculations have been carried out, e.g., by Ruden \& Lin (1986); Bell et al. (1997); Gail (2001, and further references cited therein). With the exception of Gail (2001) all other authors use analytic approximations to the (Rosseland mean) extinction coefficient of the disk material. Here, the situation is most unsatisfactory and must be decisively improved in the future, because it is difficult, if not impossible, to estimate the errors that arise due to the use of inconsistent extinction coefficients.

To our knowledge, global, fully 2-D models of preplanetary accretion disks with axial symmetry have not yet been discussed in the published literature. In this paper we present very first results pertaining to the mechanical, thermal, and chemical structure and evolution of such disks which are considered to be the precursory sites of planetary formation. The guideline of our approach is to preserve as much consistency as possible when modelling the hydrodynamical, chemical, transport/mixing processes and their mutual interactions in preplanetary disks. This is the only way to interpret properly the exciting findings of recent space missions like Star Dust or Deep Impact - clear imprints of a past hot chemistry conserved in the cold cometary environment - and to arrive at a coherent picture of how, where, when, and to which extent, mass transport takes place in preplanetary nebulae.

The paper is organized as follows: Sect. 2 exhibits the set of our model equations. For the sake of definiteness, we rewrite them at full length with cylindrical $(r, z)$-coordinates. Section 3 contains a compilation and discussion of the material functions (viscosity, equation of state, chemical network, opacity). In Sects. 4 and 5 we outline the numerical strategy which we adopted to conduct our calculations and present the results, respectively. The final Sect. 6 is devoted to final remarks on the most conspicuous achievements and to a short outlook for further investigations.

\section{Basic equations}

We write our basic model equations expressing the conservation laws of physics in cylindrical coordinates. With axial symmetry the two independent spatial coordinates of the position vector are the radius $r$ and the vertical height $z$. Denoting by $u_{r}$ and $u_{z}$ the velocity components in the radial and vertical direction, respectively, the continuity equation which expresses bulk mass conservation then reads

$\frac{\partial \rho}{\partial t}+\frac{1}{r} \frac{\partial}{\partial r}\left(r \rho u_{r}\right)+\frac{\partial}{\partial z}\left(\rho u_{z}\right)=0$.

Protoplanetary "fluids" are necessarily multi-component flows consisting of many gaseous species and microscopically small solid dust particles. In different parts of the disk the concentrations of the various species will differ due to chemical reactions and other processes like combustion of soot particles or condensation and evaporation of other dust particles (silicates). Both advective transport and diffusive mixing therefore play a fundamental role in the evolution of protostellar disks. Hence, in addition to the continuity Eq. (1), for each species, an advectiondiffusion equation with source term of the type

$$
\begin{aligned}
\frac{\partial}{\partial t}\left(\rho c_{i}\right)+ & \frac{1}{r} \frac{\partial}{\partial r}\left(r \rho u_{r} c_{i}\right)+\frac{\partial}{\partial z}\left(\rho u_{z} c_{i}\right)= \\
& \frac{1}{r} \frac{\partial}{\partial r}\left(r \rho D \frac{\partial c_{i}}{\partial r}\right)+\frac{\partial}{\partial z}\left(\rho D \frac{\partial c_{i}}{\partial z}\right)+R_{i},
\end{aligned}
$$

holds. $c_{i}, R_{i}$ are the concentration per weight $\left(\sum_{i} c_{i}=1\right)$ and the source or sink $\left(\sum_{i} R_{i}=0\right)$ of the $i$ th species, respectively, and $D$ is the diffusion coefficient. There is a qualitative relation between the (turbulent) kinematic viscosity, $v_{\text {turb }}$ (cf. Sect. 3.1), and the diffusion coefficient, $D$, which is expressed by the Schmidt number,

$\mathcal{S}:=\frac{v_{\text {turb }}}{D}$

$\mathcal{S}$ is expected to be of the order of unity. In a recent local study of turbulent mixing processes in the outer solar nebula, Turner et al. (2006) suggest that the widely adopted "canonical" value, $\mathcal{S}=$ 1 , would indeed be a realistic choice. This finding is based on 3-D MHD calculations in the shearing-box approximation with vertical stratification. However, whether or not results of such purely local investigations are really representative for the global mixing patterns in the disk is an open question.

Large Schmidt numbers indicate substantially reduced diffusive mixing, that is, $D \rightarrow 0$ if $\mathcal{S} \rightarrow \infty$. In this asymptotic limit, advection is the only mechanism to drive transport and mixing of the disk material.

As long as relative flow velocities between the various species - in particular, the dust grains - are either constant or sufficiently small, conservation of momentum is expressed by the minimum set of the three equations of motion in the radial and vertical direction (linear momentum) and in the azimuthal direction (angular momentum). Dust particles of (sub-)micron size which we are dealing with are strongly coupled to the gas and, hence, will exhibit only negligibly small drift velocities.

If $\Phi$ denotes the gravitational potential, $\eta:=\rho v_{\text {turb }}$ the dynamical viscosity, and $u_{\phi}$ the azimuthal velocity, the NavierStokes equations in the radial and vertical direction are given in explicit form as

$$
\begin{gathered}
\frac{\partial}{\partial t}\left(\rho u_{r}\right)+\frac{1}{r} \frac{\partial}{\partial r}\left(r \rho u_{r} u_{r}\right)+\frac{\partial}{\partial z}\left(\rho u_{z} u_{r}\right)= \\
\rho\left(\frac{u_{\phi}^{2}}{r}-\frac{\partial \Phi}{\partial r}\right)-\frac{\partial P}{\partial r}+\frac{\rho \kappa}{c} F_{r} \\
+\frac{4}{3} \frac{\partial}{\partial r}\left[\eta r \frac{\partial}{\partial r}\left(\frac{u_{r}}{r}\right)\right]+\frac{2}{3} \frac{\partial}{\partial r}\left[\eta\left(\frac{u_{r}}{r}-\frac{\partial u_{z}}{\partial z}\right)\right] \\
+2 \eta \frac{\partial}{\partial r}\left(\frac{u_{r}}{r}\right)+\frac{\partial}{\partial z}\left[\eta\left(\frac{\partial u_{r}}{\partial z}+\frac{\partial u_{z}}{\partial r}\right)\right]
\end{gathered}
$$

and

$$
\begin{gathered}
\frac{\partial}{\partial t}\left(\rho u_{z}\right)+\frac{1}{r} \frac{\partial}{\partial r}\left(r \rho u_{r} u_{z}\right)+\frac{\partial}{\partial z}\left(\rho u_{z} u_{z}\right)= \\
-\rho \frac{\partial \Phi}{\partial z}-\frac{\partial P}{\partial z}+\frac{\rho \kappa}{c} F_{z} \\
+\frac{1}{r} \frac{\partial}{\partial r}\left[r \eta\left(\frac{\partial u_{r}}{\partial z}+\frac{\partial u_{z}}{\partial r}\right)\right] \\
+\frac{2}{3} \frac{\partial}{\partial z}\left[\eta\left(2 \frac{\partial u_{z}}{\partial z}-\frac{1}{r} \frac{\partial}{\partial r}\left(r u_{r}\right)\right)\right]
\end{gathered}
$$


respectively. Though of minor importance for preplanetary accretion disks, the radiation acceleration terms, $\rho \kappa F_{r} / c$ and $\rho \kappa F_{z} / c$, are included in the respective Navier-Stokes Eqs. (4) and (5) for the radial and vertical direction. $\kappa$ is the (grey) extinction coefficient, $c$ the speed of light. In principle, the vector of the radiative force is given via the integral of the product of the (nongrey) extinction coefficient, $\kappa_{v}$, times the radiative flux, $\boldsymbol{F}_{\text {rad, } v}$ over all frequencies, $v$, i.e., the expression $(1 / c) \int_{0}^{\infty} \kappa_{v} \cdot \boldsymbol{F}_{\text {rad, },} \mathrm{d} v$ is the radiation pressure force per unit mass, with $\boldsymbol{F}_{\mathrm{rad}, v}=$ $\left(F_{r, v}, 0, F_{z, v}\right)$ having non-trivial components only in the $r$ - and $z$-direction by virtue of the assumed axial symmetry.

The third Navier-Stokes equation relates to the conservation of angular momentum:

$$
\begin{array}{r}
\frac{\partial}{\partial t}\left(\rho r u_{\phi}\right)+\frac{1}{r} \frac{\partial}{\partial r}\left(r \rho u_{r} r u_{\phi}\right)+\frac{\partial}{\partial z}\left(\rho u_{z} r u_{\phi}\right)= \\
\frac{1}{r} \frac{\partial}{\partial r}\left[r^{3} \eta \frac{\partial}{\partial r}\left(\frac{u_{\phi}}{r}\right)\right]+\frac{\partial}{\partial z}\left[\eta \frac{\partial}{\partial z}\left(r u_{\phi}\right)\right] .
\end{array}
$$

For the sake of completeness, if necessary, the self-gravitation of the disk material is taken into account by solving Poisson's equation

$$
\frac{1}{r} \frac{\partial}{\partial r}\left(r \frac{\partial \Phi_{\mathrm{disk}}}{\partial r}\right)+\frac{\partial^{2} \Phi_{\mathrm{disk}}}{\partial z^{2}}=4 \pi G \rho,
$$

where $G$ is the gravitational constant and $\Phi_{\text {disk }}$ denotes the gravitational potential of the disk. The total gravitational potential, $\Phi$, appearing in the Navier-Stokes Eqs. (4) and (5) is the sum of both the central protosun's and the disk's potential, that is, $\Phi=\Phi_{\text {sun }}+\Phi_{\text {disk }}$.

Two energy balance equations, one for the matter and the other one for the radiation, together with the equations of state, complete the set of the basic equations. Let $\varepsilon, \varepsilon_{\text {rad }}$ be the specific internal energy of the disk material and the radiation energy density, respectively, $\boldsymbol{F}_{\text {rad }}:=\left(F_{r}, F_{\phi} \equiv 0, F_{z}\right)$ the (total) radiation flux integrated over all frequencies, $\kappa_{v}$ the frequencydependent extinction coefficient, and $a_{v}, 0 \leq a_{v} \leq 1$, the albedo. Both $\kappa_{v}$ and $a_{v}$ depend, in general, on the frequency of the radiation field, $I_{v}(t, \boldsymbol{x}, \boldsymbol{k})$, which is a function of the time, $t$, the spatial position, $\boldsymbol{x}$, and the direction of the wave vector (=unit vector), $\boldsymbol{k}$. However, in this study, we will deal exclusively with the widely used, but rather crude, approximation of the frequencyindependent (grey) radiative transfer. To this end, appropriate average values of the extinction coefficient, $\kappa_{v}$, and the "true absorption", $\kappa_{v}^{\mathrm{abs}}:=\kappa_{v}\left(1-a_{v}\right)$, are adopted. In fact, in the calculations so far carried out, we go even further and neglect scattering, i.e., $a_{v} \equiv 0$, which is not too bad an approximation in the low temperature regime $(T \lesssim 2000 \mathrm{~K})$, and take the Rosseland mean, $\kappa_{\mathrm{R}}$, of the absorption coefficient plainly as the effective opacity, $\kappa:=\kappa_{\mathrm{R}}$.

For writing down the equations which couple the thermal and radiative energy, it is, above all, more instructive and transparent to retain the basic frequency-dependent quantities in the respective terms rather than to start with frequency-integrated quantities right from the beginning. In so doing - for the simplest case of isotropic scattering - we start out with the source function of radiation, $S_{v}$, which is the sum of the albedo-weighted thermal emission and pure scattering, that is,

$S_{v}=\left(1-a_{v}\right) \cdot B_{v}(T)+a_{v} \cdot J_{v}$, where $B_{v}(T)$ is the Planck function at the temperature, $T$, and $J_{v}$ denotes the zeroth moment of the radiation field, $I_{v}(t, \boldsymbol{x}, \boldsymbol{k})$, thus $J_{v}(t, \boldsymbol{x})=(1 / 4 \pi) \oint_{4 \pi} I_{v}(t, \boldsymbol{x}, \boldsymbol{k})|\mathrm{d} \boldsymbol{k}|$. Furthermore,

$\varepsilon_{\mathrm{rad}}=\frac{4 \pi}{c} J, \quad J:=\int_{0}^{\infty} J_{v} \mathrm{~d} v$

is the (total) radiation energy density. Analogously, the first and the second moment, $\boldsymbol{H}_{v}:=(1 / 4 \pi) \oint_{4 \pi} I_{v} \cdot \boldsymbol{k}|\mathrm{d} \boldsymbol{k}|$ and $\mathrm{K}_{v}:=$ $(1 / 4 \pi) \oint_{4 \pi} I_{v} \cdot(\boldsymbol{k} \otimes \boldsymbol{k})|\mathrm{d} \boldsymbol{k}|$, respectively, relate to the radiation flux vector, $\boldsymbol{F}_{\text {rad }}$, and the radiation pressure tensor, $\mathrm{P}_{\text {rad }}$, by

$\boldsymbol{F}_{\mathrm{rad}}=4 \pi \boldsymbol{H}, \quad \boldsymbol{H}:=\int_{0}^{\infty} \boldsymbol{H}_{v} \mathrm{~d} v$

and

$\mathrm{P}_{\mathrm{rad}}=\frac{4 \pi}{c} \mathrm{~K}, \quad \mathrm{~K}:=\int_{0}^{\infty} \mathrm{K}_{v} \mathrm{~d} v$.

By introducing the frequency-integrated source function (exactly valid for vanishing albedo, $a_{v} \equiv 0$ ),

$S:=\int_{0}^{\infty} S_{\nu} \mathrm{d} v=\frac{\sigma}{\pi} T^{4}$

( $\sigma$ denotes Stefan-Boltzmann's constant) the first law of thermodynamics governing the balance of the internal energy is expressed as

$$
\begin{array}{r}
\frac{\partial}{\partial t}(\rho \varepsilon)+\frac{1}{r} \frac{\partial}{\partial r}\left(r \rho u_{r} \varepsilon\right)+\frac{\partial}{\partial z}\left(\rho u_{z} \varepsilon\right)+P\left[\frac{1}{r} \frac{\partial}{\partial r}\left(r u_{r}\right)+\frac{\partial u_{z}}{\partial z}\right]= \\
4 \pi \rho \kappa(J-S)+\dot{Q}_{\text {vis }}+\dot{Q}_{\text {chem }}
\end{array}
$$

The source term on the right hand side consists of three contributions, the radiation term, the energy dissipation term, $\dot{Q}_{\text {vis }}$, due to (turbulent) viscosity and/or shock waves, and the "thermochemical" term, $\dot{Q}_{\text {chem }}$, arising from the chemical reactions. Note that exothermal and endothermal reactions are, by definition, characterized by $Q_{\text {chem }}<0$ and $Q_{\text {chem }}>0$, respectively, so that the rate of chemical heat, $\dot{Q}_{\text {chem }}$, released/consumed per unit volume reads

$\dot{Q}_{\text {chem }}=-\sum_{\text {all reactions, } r} k_{i, j[, l]}^{(r)}(T) n_{i} n_{j}\left[n_{l}\right] Q_{\text {chem }}^{(r)}$.

Here, $k_{i, j[, l]}^{(r)}(T)$ denotes the temperature-dependent rate constant of the $r$ th reaction (three-particle-reactions are indicated by square brackets) with $n_{i}, n_{j},\left[n_{l}\right]$ being the respective number densities of the reacting species and $Q_{\text {chem }}^{(r)}$ the chemical heat per reaction (cf. Sect. 3.3.1, Eq. (24), and Table 4). The two (algebraic) equations of state

$P=P(\rho, T), \quad \varepsilon=\varepsilon(\rho, T)$

relate the gas pressure, $P$, and the internal energy, $\varepsilon$, with the mass density, $\rho$, and the temperature, $T$, respectively.

We have restricted ourselves to the Eddington approximation, that is, with $\mathrm{E}$ denoting the unit tensor, we have set $\mathrm{K}=$ $(J / 3) E$ in our simulations. Expressed with cylindrical coordinates, the balance equation for the radiative energy density, $\varepsilon_{\mathrm{rad}}$, being proportional to $J$ according to Eq. (9), then reads (see, e.g., Castor 1986) in explicit form

$$
\begin{array}{r}
\frac{\partial J}{\partial t}+\frac{1}{r} \frac{\partial}{\partial r}\left(r u_{r} J\right)+\frac{\partial}{\partial z}\left(u_{z} J\right)+\frac{J}{3}\left[\frac{1}{r} \frac{\partial}{\partial r}\left(r u_{r}\right)+\frac{\partial u_{z}}{\partial z}\right] \\
+c\left[\frac{1}{r} \frac{\partial}{\partial r}\left(r H_{r}\right)+\frac{\partial H_{z}}{\partial z}\right]+c \rho \kappa(J-S)=0
\end{array}
$$


$H_{r}$ and $H_{z}$ refer to the radial and vertical component of the first moment of the radiation field, respectively. By definition of axial symmetry, the azimuthal component, $H_{\phi}$, vanishes identically. Then, the Eddington approximation is expressed as

$H_{r}=-\frac{1}{3 \rho \kappa} \frac{\partial J}{\partial r}, \quad H_{z}=-\frac{1}{3 \rho \kappa} \frac{\partial J}{\partial z}$.

In case the "Eddington-flux" as defined by Eq. (16) above formally exceeds the radiative energy, we introduce an appropriate limiting factor, $0<\theta \leq 1$, to make sure that the inequality $|\boldsymbol{H}| \leq \theta \cdot J$ holds everywhere. To stay on the safe side, we have set $\theta=1 / 3$ throughout.

Aside from model-dependent initial and boundary conditions (cf. Sect. 4 for details), the system of Eqs. (1)-(6), (13), (15)-(16), and the equations of state (14) plus the other constitutive relations, e.g., the opacity and the rate coefficients for the chemical reactions (cf. Sect. 3 for details) is now complete.

\section{Constitutive relations}

It has already been emphasized that, in addition to the system of partial differential equations expressing the usual conservation of mass, momentum, and energy, a series of functional relations between the physical quantities must be taken into consideration. They characterize the material properties and are necessary to close the model equations. These "material functions" pertain to the particular choice of the "turbulent" viscosity coefficient, the equations of state (14), the rate constants of the chemical reaction network, the vapor pressure of condensable species, and the opacity.

\subsection{Viscosity}

Duschl et al. (2000) have shown that the classic Shakura \& Sunyaev (1973) $\alpha$-prescription for the "turbulent" viscosity coefficient leads to a fundamental physical inconsistency when the self-gravitation of the (stationary) disk is taken into account. The authors propose a viscosity that should depend exclusively on purely mechanical quantities. This assumption is a necessary condition to avoid inconsistent physics and its validity is further supported by the idea that the turbulence is driven essentially by the (quasi-)Keplerian shear flow in accretion disks and not so much by their thermal structure.

However, with regard to the great success of the $\alpha$-disk models in other fields of accretion disk theory, e.g., cataclysmic variables, any other simple one-parameter viscosity model should in some way or another approach the standard $\alpha$-model for vanishing self-gravitation. According to Duschl et al. (2000) this requirement can be fulfilled if the coefficient of the "turbulent" dynamic viscosity, $\eta$, takes on the form

$\eta:=\rho \cdot \beta \cdot r^{2} \Omega$,

where $\beta$ is a free parameter scaling with the inverse of the "critical" Reynolds-number, $\mathrm{Re}_{\mathrm{cr}}$, which is expected to be in the range $10^{2 \ldots 4}$. Hence, we may justly define

$\beta:=\operatorname{Re}_{\mathrm{cr}}{ }^{-1} \simeq 10^{-4 \ldots-2}$.

A comparison with the $\alpha$-prescription yields, after some simple algebra, the relation

$\beta=\alpha \cdot\left(\frac{h}{r}\right)^{2}$
Thus, the $\beta$-viscosity is even identical with the classic $\alpha$-viscosity if the disk's half-thickness, $h:=c_{\mathrm{S}} / \Omega$, with $c_{\mathrm{s}}$ and $\Omega:=u_{\phi} / r$ being the speed of sound and the Keplerian angular velocity, respectively, exhibits a strictly linear dependence on the radial distance, $r$. Moreover, if we restrict ourselves to the so-called dissipation limit by demanding that the turbulent velocities must not exceed the speed of sound, it can be shown that the more general $\beta$-prescription includes the more familiar $\alpha$-prescription as a special case in the limit of negligible selfgravity. Again, details are given in Duschl et al. (2000).

The $\beta$-prescription for the turbulent viscosity is an important generalization of the classic $\alpha$-prescription. Already for apparently low-mass disks containing only a few percent of the central mass, $M_{\star}$, that is, if the inequality $M_{\text {disk }} / M_{\star} \gtrsim 0.03 \ldots 0.05$ holds, the disk's self-gravity in the vertical direction becomes comparable to, or greater than, the vertical component of the central star's gravitational acceleration. In general, the transition takes place for disk masses well below the limit at which selfgravitation affects the radial disk structure, too. In that sense "moderately" self-gravitating disks will still very nearly show Keplerian rotation and may be referred to as self-gravitating Keplerian accretion disks. It is our opinion that preplanetary nebulae belong to this "intermediate" category of self-gravitating disks, at least during their initial evolutionary stages.

This is the reason why we have favored the $\beta$-prescription from the very beginning, although we have not yet implemented an appropriate Poisson solver in the code. This will be done in the next step. In our calculations we have set $\beta=10^{-3}$ throughout.

\subsection{Equation of state}

The main constituents of the material in preplanetary disks are molecular hydrogen and helium $(\approx 98 \%$ per weight, cf. Table 2$)$. For the temperature and density range $(T \gtrsim 20 \mathrm{~K}, \rho \lesssim$ $10^{-8} \mathrm{~g} \mathrm{~cm}^{-3}$ ) we are dealing with here, the disk material can be treated as an ideal gas. If $n_{i}$ denotes the number density of the $i$ th species, $i=1, \ldots, L$, and $T$ the temperature, then the gas pressure, $P$, is given by the sum of the partial pressures, $P_{i}=n_{i} \cdot k_{\mathrm{B}} T$, thus

$P=\sum_{i=1}^{L} P_{i}=\sum_{i=1}^{L} n_{i} \cdot k_{\mathrm{B}} T$.

Introducing the mean molecular weight by

$\mu:=\frac{\sum_{i=1}^{L} A_{i} n_{i}}{\sum_{i=1}^{L} n_{i}}=\frac{1}{\sum_{i=1}^{L}\left(c_{i} / A_{i}\right)}$,

where $A_{i}$ and $c_{i}$ are the molecular weight of the $i$ th species and its concentration by weight, respectively, the equation of state reads

$P=\frac{\mathcal{R}}{\mu} \rho T, \quad \mathcal{R}=k_{\mathrm{B}} \cdot \mathcal{A}$,

where $\mathcal{R}$ and $k_{\mathrm{B}}$ are the universal gas and Boltzmann's constant, respectively, and $\mathcal{A}$ denotes Avogadro's number.

To calculate the internal energy of the gas, $\varepsilon$, we have assumed that the ratio of the specific heats, $\gamma:=c_{p} / c_{v}$, at constant pressure and volume is a specific constant for every species depending on whether it exists as a monatomic, diatomic, or polyatomic molecule with 3,5 , and 6 degrees of freedom, that is, $\gamma=5 / 3,7 / 5$, and $4 / 3$, respectively. This is not too bad an approximation for moderate temperatures, say, in between 
$150 \ldots 200 \mathrm{~K}$ and $1300 \mathrm{~K}$, where the rotational degrees of freedom are excited. The relation between the temperature, $T$, and the specific internal energy, $\varepsilon$, then is determined easily by summing up the relative contributions of the various species to the internal energy according to their abundance, $c_{i}, i=1, \ldots, L$. This yields the desired relation

$\varepsilon=\mathcal{R} T \cdot \sum_{i=1}^{L} \frac{c_{i}}{A_{i}\left(\gamma_{i}-1\right)}$.

At decreasing temperatures $(\$ 150 \mathrm{~K})$, the (diatomic) hydrogen molecule - the most abundant species - starts quickly freezing in its rotational degrees of freedom and tends to behave more and more like a monatomic molecule. In fact, $\gamma$ becomes close to 5/3 already at a temperature of about $80 \mathrm{~K}$. Since we are mainly interested in the warmer regions of the preplanetary disk, we have neglected such thermodynamical subtleties as well as the action of the latent heat pertaining to the sublimation and condensation processes of, e.g., water and silicates in our current calculations. We shall remove all these relatively weak shortcomings in future simulations.

\subsection{Chemical reactions}

For the sake of simplicity, we have restricted ourselves exclusively to the gas-phase chemistry of neutral atoms and molecules consisting of hydrogen, carbon, and oxygen. These are the most abundant volatile elements which also provide the most abundant molecules in the gas phase, except for nitrogen. The nitrogen chemistry and the chemistry of less abundant elements is not yet implemented. Helium is treated as an inert species which contributes to the pressure but is not involved in the chemistry.

The chemistry does not consider ion-molecule reactions because the model calculation presented later considers the disk region between 0.8 and 5.8 AU where according to our previous results (Finocchi \& Gail 1997) the chemistry is dominated by reactions between neutral species. Also surface reactions on dust grains, in particular catalytic reactions, are not implemented (though they may be important, see the general discussion of the chemistry in the Solar Nebula by Fegley Jr. \& Prinn 1989).

Also the combustion of the microscopic graphite/soot particles have not yet been included in our explorative numerical calculations. As a consequence, we have not yet dealt with the formation of hydrocarbons, which is closely tied to the oxidation of solid carbon (soot) by the hydroxyl radical (El-Gamal 1995). Otherwise there is no theoretical constraint to extend the chemical network to arbitrary sizes except the actually available, always finite computing power.

Concerning sublimation and condensation processes, we have assumed that an equilibrium state according to the respective vapor pressure of the condensable species - water ice and magnesium silicates (forsterite) in our case - is set up instantaneously. Again, the model assumptions can and will be refined in future simulations. A way of how a more sophisticated approach can be implemented is explained by Gail (2003, and references cited therein) in a comprehensive treatise on the formation of minerals in accretion disks and stellar outflow.

\subsubsection{The chemical network}

The present network for the C-, H-, O-chemistry comprises a set of 90 gas-phase reactions between the 13 species, $\mathrm{H}, \mathrm{H}_{2}, \mathrm{O}, \mathrm{O}_{2}$, $\mathrm{OH}, \mathrm{H}_{2} \mathrm{O}, \mathrm{HO}_{2}, \mathrm{H}_{2} \mathrm{O}_{2}, \mathrm{CO}, \mathrm{CO}_{2}, \mathrm{HCO}, \mathrm{H}_{2} \mathrm{CO}$, and (gaseous) $\mathrm{C}$ from the elements $\mathrm{H}, \mathrm{O}$, and $\mathrm{C}$. Figure 1 illustrates the network

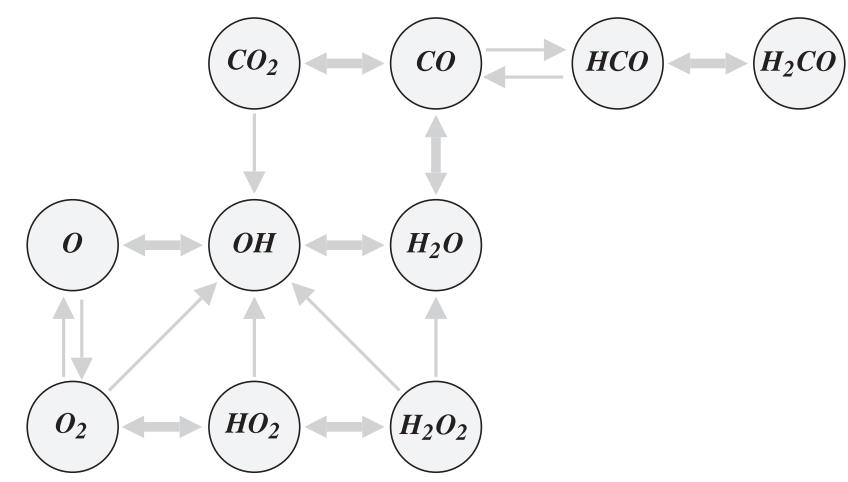

Fig. 1. Schematic representation of the reaction network considered in the present work.

in a schematic way. This set of chemical compounds containing hydrogen, carbon, and oxygen comprises the most abundant molecules, $\mathrm{H}_{2}, \mathrm{H}_{2} \mathrm{O}, \mathrm{CO}$, and $\mathrm{CO}_{2}$, radicals (e.g., $\mathrm{OH}$ ) and the respective free atoms, according to a standard cosmic element mixture. Because of the aforementioned reasons, hydrocarbons are not yet included.

From the above set of atomic and molecular species, five of them, i.e., $\mathrm{H}, \mathrm{H}_{2}, \mathrm{H}_{2} \mathrm{O}, \mathrm{OH}$, and $\mathrm{CO}$ enter into the calculation of the opacity coefficient for higher temperature regions in the disk, where the refractory dust grains evaporate (cf. Sect. 3.4). This subset of species covers the atomic and molecular species that are the most important sources of extinction in the dust-free region of the disk (cf. Fig. 7 of Alexander \& Ferguson 1994) see also Ferguson et al. (2005) - except perhaps for $\mathrm{TiO}$ which, however, is not the dominating sources of opacity for the still relatively low temperatures $(<1500 \mathrm{~K})$ in our disk model.

Methane, $\mathrm{CH}_{\mathbf{4}}$, may contribute to the gas-phase opacity at temperatures $<1800 \mathrm{~K}$ as it does in Brown Dwarf atmospheres (cf. Geballe et al. 2002), but the total opacity at low temperatures is dominated by dust, and methane has not proved to be an important opacity source, for instance, in the opacity calculations of Ferguson et al. (2005). Likewise, neglecting nitrogen compounds as possible contributors to the gas opacity is uncritical. This is because in the oxygen-rich environment only $\mathrm{N}_{2}$ is abundant, however, molecular nitrogen does not contribute to the opacity. Other N-bearing compounds are rare and are at best minor opacity sources in an oxygen-rich element mixture.

Despite the present limitation of the chemistry of the gas phase to a rather small set of species this already accounts for the full non-linear coupling between chemistry, radiative transfer, the temperature structure of the disk, and the disks hydrodynamic evolution, because all important gas phase opacity sources are already included. Any future extension of the set of species considered in the calculation of the gas phase chemistry will not add any new aspects to the problem of disk structure and evolution since no important new opacity sources will be added by this. The only major shortcoming may be that the different thermal behavior of ortho- and para- $\mathrm{H}_{2}$ at temperatures of the order of $100 \mathrm{~K}$ and less is not considered in the model since presently the chemistry does not discriminate between ortho- and para- $\mathrm{H}_{2}$.

We allow for reactions between two and at most three species. The 90 respective temperature-dependent rate constants, $k=k_{i j[l]}(T), i, j, l=1, \ldots, 13$, are assumed to be expressible in terms of the three so-called Arrhenius parameters, $A, b$, $T_{0}$, that is,

$k=A \cdot T^{b} \cdot \exp \left(-T_{0} / T\right)$. 
The Arrhenius parameters and the amount of heat, $Q_{\text {chem }}$, released/consumed by the respective exothermal $\left(Q_{\text {chem }}<0\right)$ or endothermal $\left(Q_{\text {chem }}>0\right)$ reaction are listed in Table 4 . The numbers are compiled from various sources that are given at the end of the table.

The net change of the number density, $n_{i}$, of the $i$ th species per unit time results from balancing the individual constructive and destructive reactions that raise and diminish, respectively, the value of $n_{i}$. Thus, the following set of 13 ordinary differential equations for each of the 13 species listed above holds:

$$
\begin{aligned}
\frac{\mathrm{d} n_{i}}{\mathrm{~d} t}= & \sum_{j, l=1}^{13} k_{j l}^{(\rightarrow i)}(T) n_{j} n_{l}+\sum_{j, l, m=1}^{13} k_{j l m}^{(\rightarrow i)}(T) n_{j} n_{l} n_{m} \\
& -\sum_{j=1}^{13} k_{i j}^{(i \rightarrow)}(T) n_{i} n_{j}-\sum_{j, l=1}^{13} k_{i j l}^{(i \rightarrow)}(T) n_{i} n_{j} n_{l} ; \\
& i=1, \ldots, 13 .
\end{aligned}
$$

The label " $\rightarrow i$ " indicates constructive reactions that build up the $i$ th species, " $i \rightarrow$ " marks destructive reactions. The solution of the system of Eqs. (24) yields, at the fixed density, $\rho=\sum_{\text {species }}^{\text {all }} A_{i} n_{i} / \mathcal{A}$, both the change of the species concentrations, i.e., the source term $R_{i}$ on the right-hand side of Eq. (2), and the accompanied net rate of the reaction heat, $\dot{Q}_{\text {chem }}$, with time, regarded as a function of the (variable) temperature. In this sense, the right-hand sides of the equation of state, (21) and (22), are then known functions of the two state variables, density and temperature, which are for their part governed by the balance equations for the mass (Eq. (1)) and energy (Eqs. (13) and (15)), respectively. The numerical strategy based on the method of operator splitting (see Sect. 4.1) is perfectly adapted to transform these theoretical considerations into an efficient and robust computer code.

\subsubsection{Sublimation and condensation processes}

Among the many volatile species in the gas phase, which can condense out to form "ice" mantles on the refractory grains (silicates, soot/graphite, ...), e.g., $\mathrm{H}_{2} \mathrm{O}, \mathrm{CO}, \mathrm{CO}_{2}, \mathrm{NH}_{3}$, only to mention the most abundant molecules, up to now, we have only taken the evaporation/condensation of water $\left(\mathrm{H}_{2} \mathrm{O}\right)$ ice and silicates, however rather schematically, into account. It is assumed that the partial pressure of the condensable species in the gas phase amounts at most to its vapor pressure. Any surplus molecules of this species are regarded to belong to the condensed phase.

Water ice. This is assumed to condense on top of the existing dust grains of any kind. At the low pressures typical for preplanetary disks in the region at several AU distance from the star water ice condenses at temperatures of about $150 \mathrm{~K}$. The vapor pressure of $\mathrm{H}_{2} \mathrm{O}$ over water ice at low temperatures (in units dyn $\mathrm{cm}^{-2}$ ) is calculated from the analytical interpolation formula of Lichtenegger \& Kömle (1991),

$$
\begin{aligned}
\log _{10}\left(P_{\mathrm{eq}}^{\mathrm{H}_{2} \mathrm{O}}\right)= & -2445.6 / T+8.23 \log _{10}(T)-3.632 \\
& -T\left(1.677 \times 10^{-2}-1.205 \times 10^{-5} T\right) .
\end{aligned}
$$

The fraction $f_{\text {ice }}$ of the water vapor condensed into ice is given by

$f_{\text {ice }}=\max \left(1-\left(P_{\mathrm{eq}}^{\mathrm{H}_{2} \mathrm{O}} / P^{\mathrm{H}_{2} \mathrm{O}}\right), 0\right)$,

where $P^{\mathrm{H}_{2} \mathrm{O}}$ corresponds to the totally available $\mathrm{H}_{2} \mathrm{O}$.
Silicates. The treatment of silicate evaporation is somewhat complicated since silicates decompose on evaporation (cf. Gail 2003). Since solid iron disappears by evaporation under the pressure conditions prevailing in a pre-planetary disk at almost the same temperature as forsterite $\left(\mathrm{Mg}_{2} \mathrm{SiO}_{4}\right)$ - the last stable of the main silicate dust components upon heating - we calculate the degree of condensation of iron and take this as a substitute for the degree of condensation $f_{\mathrm{d}}$ of silicate dust.

The equilibrium pressure $P_{\mathrm{eq}}^{\mathrm{Fe}}$ of iron (Fe) (in units dyn $\mathrm{cm}^{-2}$ ) is calculated from the analytical expression

$\log _{10}\left(P_{\text {eq }}^{\mathrm{Fe}}\right)=-21340.7 / T+13.7781-2.45567 \times 10^{-4} T$.

This follows from the expressions for the free enthalpy of formation of solids given in Sharp \& Huebner (1990). The degree of condensation is given by

$f_{\mathrm{d}}=\max \left(1-\left(P_{\mathrm{eq}}^{\mathrm{Fe}} / \epsilon_{\mathrm{Fe}} 2 P_{\mathrm{H}_{2}}\right), 0\right)$.

With respect to condensation of silicates there arises the problem that some kind of seed particle is required. While this poses no problem for ice condensation since at the low temperatures of ice condensation in any case some kind of dust is present which may serve as condensation center, this is not automatically guaranteed for the high temperature condensates. We will assume in this explorative calculation that there exist still more refractory "seeds" consisting, e.g., of solid titanium oxide or some other extremely refractory compounds, onto which silicates will be able to condense out again. A much more involved situation would emerge if such seeds were completely absent and effective nucleation processes had to be identified. The problem of calculating realistic nucleation rates is presently not yet solved and recourse to rather crude approximations has to be taken in case that no seed particles from external sources are available (cf. the discussion by Patzer 2004).

\subsection{Opacity}

The effective opacity, $\kappa$, results as the sum of the contributions according to essentially three individual types of absorbers:

1. dust grains with water-ice mantles, $\kappa=\kappa_{\mathrm{d}}^{\text {ice }}$;

2. "naked" dust grains, $\kappa=\kappa_{\mathrm{d}}$;

3. gaseous species, $\kappa=\kappa_{\mathrm{g}}$.

Concerning the individual contribution of the two types of dust particles to the opacity, we adopt the canonical ${ }^{1}$ temperature dependence, that is (Bell \& Lin 1994),

$\kappa_{\mathrm{d}}^{\text {ice }}=2 \times 10^{-4} T^{2}$

and

$\kappa_{\mathrm{d}}=0.1 \sqrt{T}$

respectively. If $f_{\text {ice }}$ and $f_{\mathrm{d}}$ (with $0 \leq f_{\text {ice }}, f_{\mathrm{d}} \leq 1$ ), denote the fraction of ice-coated dust particles and "naked" dust particles, respectively, the resulting total dust opacity, $\kappa_{\mathrm{d}}^{\text {tot }}$, is calculated as

$\kappa_{\mathrm{d}}^{\text {tot }}=f_{\text {ice }} \kappa_{\mathrm{d}}^{\text {ice }}+f_{\mathrm{d}} \cdot\left(1-f_{\text {ice }}\right) \kappa_{\mathrm{d}}$.

Water ice sublimates at much lower temperatures than the refractory dust grains. This is why the sublimation of the volatile dust

\footnotetext{
${ }^{1}$ Abundance of heavier elements according to Population I, size distribution of the dust particles according to Mathis et al. (1977); see also Gail (2001).
} 
components at the so-called "snow line", which is the relatively narrow border between "cold" and "warm" zones of the disk where water is frozen out on the silicate grains or is sublimated in the form of water vapor, respectively, is also spatially well separated from the region where the refractory particles evaporate. So, in Eq. (30), we can always assume $f_{\text {ice }} \equiv 0$ when $f_{\mathrm{d}}<1$.

As long as a population of dust grains exists the contribution of the gaseous species to the effective opacity is negligibly small. However, if the temperature rises above 1100-1200 K the dust particles commence to decompose and, finally, will evaporate completely. Then, the only sources of opacity are the remaining molecules in the gaseous state. Molecular hydrogen, the most abundant species, is only a weak absorber. Other molecules, for instance carbon monoxide and water vapor, are more efficient absorbers, but compared to dust their opacity is always relatively small. As a consequence, the disappearance of dust grains opens a deep "opacity gap" where the opacity drops several orders of magnitude (more details are given in Gail 2001).

In our calculations, we have included the contribution of the four molecules, $\mathrm{H}_{2}, \mathrm{OH}, \mathrm{H}_{2} \mathrm{O}, \mathrm{CO}$, to the gas opacity,

$\kappa_{\mathrm{g}}=\kappa_{\mathrm{H}_{2}}+\kappa_{\mathrm{OH}}+\kappa_{\mathrm{H}_{2} \mathrm{O}}+\kappa_{\mathrm{CO}}$

According to Keeley (1970) and Marigo (2002) these various contributions can be approximated in terms of the number densities, $n$, of the molecules and the temperature, $T$ (with the abbreviation $T_{4}:=10^{-4} T$ ), as follows:

$$
\begin{aligned}
\rho \cdot \kappa_{\mathrm{H}_{2}}= & \left(n_{\mathrm{H}}+n_{\mathrm{H}_{2}}\right) \cdot \frac{5.55 \times 10^{-27} T_{4}^{4}}{1+10 T_{4}^{6}+3.42 \times 10^{-5} / T_{4}^{6}} \\
\rho \cdot \kappa_{\mathrm{OH}}= & n_{\mathrm{OH}} \cdot \frac{1.4 \times 10^{-21} T_{4}^{6}}{0.1+T_{4}^{6}} \\
\rho \cdot \kappa_{\mathrm{H}_{2} \mathrm{O}}= & \left(1-f_{\text {ice }}\right) n_{\mathrm{H}_{2} \mathrm{O}} \cdot\left[\frac{2.6 \times 10^{-27}}{4.23 \times 10^{-4}+T_{4}^{4}}\right. \\
& \left.+\frac{9.72 \times 10^{-21}}{1+3780 T_{4}^{10}} \exp \left(-\frac{3.2553}{0.37+T_{4}}\right)\right] \\
\rho \cdot \kappa_{\mathrm{CO}}= & n_{\mathrm{CO}} \cdot 2.75 \times 10^{-26} .
\end{aligned}
$$

By combining Eqs. (28)-(32) we end up with the final expression

$\kappa=\kappa_{\mathrm{d}}^{\mathrm{tot}}+\kappa_{\mathrm{g}}$

for the effective opacity, $\kappa$. It is evident that the value of $\kappa$, derived in the straightforward manner as indicated above, is only a very crude approximation to the true Rosseland mean of the absorption coefficient we ought to use in our calculations. Within the framework of $(1+1)$-D models, an important step to overcome this unsatisfactory situation has been made by Gail (2001) in his successful attempt to treat self-consistently, by assuming chemical equilibrium, the condensation and vaporization of the minerals that dominate the absorption properties of the disk material. How to derive reliable numbers concerning the optical properties of (generally many-layered) ice-coated dust particles and, even more intriguing, their agglomerates, is still an open problem (see, however, Voshchinnikov et al. 2005, 2006). With regard to our simplified treatment of radiative energy transfer (Eddington approximation) we have restricted ourselves, for the time being, to the approximate determination of the opacity, $\kappa$, according to Eq. (33) above.

\section{Numerical method}

To begin with, it may be illustrative to make a few general statements about why there is a need to consider spatially fully 2-D and, eventually, 3-D models of protoplanetary disks.

Simplified radially 1-D, vertically one-zone models or more elaborate $(1+1)$-D have proven to be very powerful for exploring the overall features of disk evolution. With these approximations, the basic effects of turbulence within the disk, comprising both an efficient redistribution of angular momentum and diffusive radial mixing of the disk material, can be well studied. However, there remain specific shortcomings which can be removed only if the assumption of the disk to be strictly hydrostatic in the vertical direction is abandoned. For instance, within the framework of the $(1+1)-\mathrm{D}$ disk models it is, by definition, impossible to determine the real structure of the disk's velocity field, e.g., its dependence on the vertical coordinate, $z$, nor can mixing processes in the $z$-direction be described adequately: only a $z$-averaged radial net "drift" of material - in the outward or inward direction, depending on the radial distance from the center - is available. In particular, the investigation of detailed advective mass transport and mixing is clearly beyond the scope of the (1+1)-D models.

Thus, in order to be able to describe the various physical and chemical processes, being effective in protoplanetary disks, in a more realistic way, honest 2-D models with axial symmetry must be taken into consideration. This means, together with solving the extensive chemical network including not only pure gas-phase reactions, but also combustion, chemi-sputtering as well as sublimation and condensation processes of microscopic dust particles, to set about an extremely computer time consuming exercise. It does not seem appropriate to aim at even more involved, fully 3-D, disk models in the first step. At this point it is worth emphasizing that, after all, we are going to present in the following the outcome of the first pilot study for fully 2-D disk models with axial symmetry.

\subsection{Numerical strategy}

Global hydrodynamical models of protoplanetary disks extending from about 0.1 astronomical units (AU) up to, say, $100 \mathrm{AU}$ would strictly demand the use of implicit numerical schemes, owing to the fact that accretion disks exhibit largely dispersed characteristic scales. This leads to prohibitively small CourantFriedrichs-Lewy (CFL-) timesteps, $\delta t_{\mathrm{CFL}}$, which set a severe constraint for the efficiency of explicit schemes. If $\Delta r_{i}:=r_{i}-r_{i-1}$, $i=1, \ldots, I$, and $\Delta z_{k}:=z_{k}-z_{k-1}, k=1, \ldots, K$ denote the spatial step sizes according to an appropriate discretisation $\left(r_{i}, z_{k}\right)_{i=0, \ldots, I ; k=0, \ldots, K}$ of the $(r, z)$-plane (see Sect. 4.1.1 below), a useful estimate of $\delta t_{\mathrm{CFL}}$ for our purposes turned out to be the expression,

$\delta t_{\mathrm{CFL}}=\min _{i, k}\left[\frac{\Delta \ell_{i, k}}{\left(c_{\mathrm{s}}\right)_{i, k}+\sqrt{\left(u_{r}^{2}+u_{z}^{2}\right)_{i, k}}+12\left(v_{\mathrm{tot}}\right)_{i, k} / \Delta \ell_{i, k}}\right]$.

As an abbreviation, we have set $\Delta \ell_{i, k}:=\min \left(\Delta r_{i}, \Delta z_{k}\right)$ and $v_{\text {tot }}:=$ $v_{\text {turb }}+v_{\text {avis }}$, being the sum of the turbulent and artificial viscosity coefficient; $c_{\mathrm{S}}$ denotes the sound velocity.

However, implicit methods, which are not subject to the restrictive CFL-condition on the timestep, are in general very costly and have been successfully applied so far only to the purely hydrodynamical aspect of accretion disks (Keller 2003).

As a compromise, mixed explicit-implicit numerical schemes seem to be best suited for simulating selected parts 
of the disk at the expense of being forced to invent welleducated, but inevitably somewhat artificial, boundary conditions. Nevertheless, there is good hope that the general numerical strategy just briefly outlined will translate into a robust computer code that could serve as the working horse for many years to come.

\subsubsection{Discretisation}

We use a finite-volume scheme and discretise the Navier-StokesEqs. (1)-(6) for compressible fluids on a staggered $(r, z)$-grid of a cylindrical coordinate system, where $(r, \phi, z)$ denotes the radial, azimuthal, and vertical coordinate, respectively. Scalar quantities are defined in the interior, the individual components of vectorial quantities are tied to the respective boundary surfaces of the numerical cells. As we have already pointed out in Sect. 3.1, turbulent viscosity is parameterized according to the $\beta$-disk prescription by Duschl et al. (2000). Self-gravitation, i.e., a suitable Poisson solver, is currently not yet implemented in the existing code.

Since we restrict ourselves to axially symmetric geometry, shock fronts in the azimuthal direction (spiral- or bar-like structures) are excluded. The artificial tensor viscosity term is tailored in a way as to smooth out shock fronts in the $(r, z)$-plane without changing the local distribution of angular momentum. A chemical network for the $\mathrm{C}$-, $\mathrm{H}$-, O-chemistry ("CHO-chemistry" for short) is implemented and the various species undergo diffusive mixing according to Eq. (2). Depending on whether the dust grains are ice-coated or not, grey opacities are calculated from respective fit formulae, the albedo is assumed to be zero. To model the (grey) radiation transport (Eq. (16)) we employ the Eddington approximation with flux delimiter (the absolute value of the 1st moment $|\boldsymbol{H}|$ must not exceed the 0th moment $J$ !). Finally, we take the two energy balance Eqs. (13) and (15) into account.

\subsubsection{Two-step solution procedure}

The equations are numerically solved by a two-step operatorsplitting procedure according to Norman \& Winkler (1986). In the first source step yielding intermediate updates of the various physical variables - such as the velocity components, concentrations, etc. - for the timestep, $\delta t<\delta t_{\mathrm{CFL}}$, only the source terms in the equations are taken into account. In the second transport step the remaining advection terms are evaluated to perform the final updating. A nominal second-order van-Leer scheme is applied to carry out the transport step.

As already mentioned, energy transport by radiation is modelled according to the Eddington approximation. The energy balance equation for the radiation field then reduces to a spatially 2-D diffusion equation with a source term that couples to the energy balance equation for the disk material. In optically thin regions, where the density (or the opacity) is low and the Eddington approximation becomes increasingly worse, the respective "radiative" CFL-timestep,

$\delta t_{\mathrm{CFL}}^{\mathrm{rad}} \simeq \frac{\min (\Delta r, \Delta z)}{c} \Delta \tau$,

would easily become much lower than the usual "hydrodynamical" CFL-timestep, $\delta t_{\text {CFL }}$, as defined in Eq. (34). In (35), the effective optical depth $\Delta \tau=\min (\Delta r, \Delta z) \kappa \rho$, and $\min (\Delta r, \Delta z) / c$ is the minimum light travel time through the numerical cell of size $\Delta r \times \Delta z$. As a consequence, the source step for the energy equation - with the mass density, $\rho$, kept constant by construction of the two-step algorithm - must be solved implicitly. The combination of a Newton-Raphson scheme combined with the GMRES algorithm to solve the linearized equation iteratively turned out to be a very efficient strategy to determine the temperature, pressure, and radiation energy density in a consistent fashion.

\subsection{Network of chemical reactions}

The Eqs. (23) of the chemical network are solved by a simple backward Euler scheme for each numerical cell independently during the source step. At that interface the code can easily be parallelized. Moreover, this nice property does not touch upon deciding whether or not a more accurate and/or efficient solver ought to be implemented.

Sublimation and condensation of water ice and silicates are outstanding test cases for the code with respect to their implications on the numerical stability of the radiative energy transport term. For the sake of simplicity, we assume equilibrium between the solid and the gas phase to be established instantaneously. In the inner, hot zones of the disk where the silicates evaporate the opacity drops abruptly several orders of magnitude, in this way giving rise to dramatic changes of the radiation field. Likewise, though to a much lesser extent, a similar situation is encountered across the "snow line", where the temperature gradients of the opacity become also very steep.

Particularly, the highly non-stationary initial "switch-on" phase, during which the biggest changes in the physical quantities take place, set the greatest standards on the numerical robustness of the code. Although the timesteps have become occasionally quite short (around 1\% of the CFL-timestep), they have never dropped to extremely small values so as to cause a lethal stop of the calculation.

\subsection{Initial conditions}

In order to test the robustness of our explicit-implicit 2-D radiation hydrodynamical code we choose an initial configuration which forces the "disk" to evolve into a pronounced dynamical regime in the first place. Only after this nonstationary "switch on" phase our disk model would asymptotically approach a quasi-stationary state that is assumed by the $(1+1)$-D description from the beginning. This situation is achieved by choosing an initially homogeneous distribution of the disk material. Table 2 contains a compilation of the initial data we used in the simulation.

We discretise the equations on a $(48 \times 64)$ Eulerian tensorproduct $(r, z)$-grid. In the $z$-direction the gridpoints $z_{j}, j=$ $0,1,2, \ldots, 64$ with $z_{0}=0 \mathrm{AU}$ are chosen to be equally spaced, i.e., $\Delta z=$ const. $\approx 0.0234 \mathrm{AU}$ (because of the assumed equatorial symmetry we need to cover only the "upper half" of the disk); the distribution of the radial gridpoints, $r_{i}, i=$ $0,1,2, \ldots, 48$ with $r_{0}=0.8 \mathrm{AU}$, is tailored according to a geometrical law. The radial mesh sizes, $\Delta r_{i}:=r_{i}-r_{i-1}, i=$ $1,2, \ldots, 48$, expand monotonically from $\Delta r_{1} \approx 0.034$ AU to $\Delta r_{48} \approx 0.235 \mathrm{AU}$ (cf. Table 2 for the actual numbers inserted).

The cosmical abundances of the chemical elements, $\mathrm{H}, \mathrm{He}$, $\mathrm{C}, \mathrm{O}, \mathrm{N}, \mathrm{Mg}, \mathrm{Si}, \mathrm{S}, \mathrm{Fe}, \mathrm{Al}$, and $\mathrm{Ca}$, according to Population I is adopted (cf. Table 1). The heavier elements, Mg, Si, S, Fe, Al, and $\mathrm{Ca}$, are tied up in solid grains (mainly in forsterite, $\mathrm{Mg}_{2} \mathrm{SiO}_{4}$, troilite, $\mathrm{FeS}$, and corundum, $\mathrm{Al}_{2} \mathrm{O}_{3}$ ) referred to as "dust" in Table 2 . In principle, $70 \%$ of the carbon would be present in the 
Table 1. Initial abundances, $\epsilon$, of the various gaseous, mantle, and grain core species relative to the total number of hydrogen atoms $\left(N_{\mathrm{H}}=1000\right)$. The initial gas temperature, $T_{\mathrm{ini}}=20 \mathrm{~K}$ (cf. Table 2$)$.

\begin{tabular}{|c|c|c|c|c|c|}
\hline \multicolumn{2}{|c|}{ gaseous } & \multicolumn{2}{|c|}{$\overline{\text { mantle }}$} & \multicolumn{2}{|c|}{ 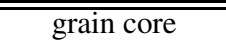 } \\
\hline species & $\epsilon$ & species & $\epsilon$ & species & $\epsilon$ \\
\hline $\mathrm{H}_{2}$ & 499.762 & $\mathrm{H}_{2} \mathrm{O}$ & 0.238 & $\mathrm{Mg}^{\frac{1}{2}}$ & 0.0720 \\
\hline $\mathrm{He}$ & 97.500 & & & $\mathrm{Si}$ & 0.0358 \\
\hline $\mathrm{CO}$ & 0.355 & & & $\mathrm{Fe}$ & 0.0324 \\
\hline $\mathrm{N}_{2}$ & 0.047 & $\left(T_{\mathrm{ini}}=\right.$ & $20 \mathrm{~K})$ & $S$ & 0.0185 \\
\hline$\left[\begin{array}{ll}\mathrm{C} & 0\end{array}\right.$ & $0.355]^{*}$ & & & $\mathrm{Al}$ & 0.0061 \\
\hline$\left[\begin{array}{ll}0 & 0\end{array}\right]$ & $0.746]^{\dagger}$ & & & $\mathrm{Ca}$ & 0.0022 \\
\hline
\end{tabular}

* Carbon is completely tied up in CO.

Oxygen is completely tied up in $\mathrm{CO}, \mathrm{H}_{2} \mathrm{O}, \mathrm{Mg}_{2} \mathrm{SiO}_{4}, \mathrm{Al}_{2} \mathrm{O}_{3}$.

Enhanced abundance of $\mathrm{Mg}$, so that $\mathrm{Si}$ is completely in forsterite $\left(\mathrm{Mg}_{2} \mathrm{SiO}_{4}\right)$.

form of condensed soot/graphite particles, with diameters typically smaller than $0.1 \mu \mathrm{m}$, but since we have not yet addressed combustion processes together with the ensuing chemistry of hydrocarbons in our calculations, we assume carbon to be already entirely transformed into (gaseous) $\mathrm{CO}$. The surplus of oxygen is tied up in water, $\mathrm{H}_{2} \mathrm{O}$. Thus, initially the gaseous component is assumed to contain only the species $\mathrm{H}_{2}, \mathrm{He}, \mathrm{N}_{2}, \mathrm{CO}, \mathrm{H}_{2} \mathrm{O}$; helium and nitrogen do not participate in the chemical reaction network. All other species listed in Sect. 3.3.1 above form later in the evolution.

\subsection{Boundary conditions}

Besides axial symmetry, we also assume symmetry of the disk with respect to the "equatorial" plane, $z=0$. There, the $z$-derivatives of the scalars like density, temperature, pressure, etc., and the vertical velocity component, $u_{z}$, must vanish. At both the inner and outer boundary we fix the radial velocity component, $u_{r}$, by taking the respective stationary drift velocity as a result of the redistribution of angular momentum according to the $\beta$-viscosity (cf. Sect. 3.1), with $\beta=10^{-3}$ in our simulation. At the inner boundary material leaks out at a (computed) total rate, $\dot{M}_{\mathrm{ib}}$, while at the outer boundary the net incoming mass flux, $\dot{M}_{\mathrm{ob}}=10^{-6} M_{\odot} \mathrm{yr}^{-1}$, is kept constant throughout the simulation. It is worth noting that there, for any height $z$, only the case of radial inflow of material is considered; it is assumed to be chemically unprocessed with a composition equal to the initially chosen one (cf. Table 2). Mass infall from "above" the disk is neglected.

The thermal boundary conditions are chosen as follows: (1) The incoming radiation from "above" is assumed to have an equivalent temperature of $20 \mathrm{~K}$. Within the framework of the Eddington approximation we have been using so far in our calculations, this radiative boundary condition is a rather crude simplification of the true situation. A more accurate description is possible, only if a more realistic radiation transport is taken into consideration. (2) There is no radiative energy transport across both the inner and the outer radial boundary. Note that the condition of neglecting radiative energy transfer in the radial direction is a basic general assumption for the $(1+1)$-D disk models; in our fully 2-D model this assumption is restricted just to the inner and outer boundary of the disk.

Further refinements of the boundary conditions could be envisaged if the structure of both the transition zone between the disk and the central star and the parent cloud in which the disk is embedded were known in more detail.
The "vacuum" density above the disk proper is given by a fixed lower limit of $\rho_{\text {vac }}=1.91 \times 10^{-15} \mathrm{~g} \mathrm{~cm}^{-3}$ in our simulation (cf. Table 2). The velocity field is artificially reset to zero after each timestep if the density shows a tendency to decrease further below this lower limit. Briefly, this - physically motivated - strategy is a simplistic way to successfully cope with a "disguised" form of a mathematically rather subtle free boundary value problem.

These particularly chosen boundary conditions allow for stationary disk models where the equality $\dot{M}_{\mathrm{ib}}=\dot{M}_{\mathrm{ob}} \equiv$ $10^{-6} M_{\odot} \mathrm{yr}^{-1}$ holds. Numerical experiments have revealed that, for practical purposes, a quiescent state, approaching the asymptotically steady state, adjusts itself already after 20-30 revolution periods of the outermost part of the disk. Complete stationarity is expected to be established only after $\beta^{-1}$ revolution periods, that is, after $1 \ldots 2 \times 10^{4} \mathrm{yr}$.

\section{Results}

We start out from a highly artificial disk configuration which is far off the mechanical equilibrium in the vertical direction (cf. Table 2 for the actually chosen initial data). Driven by the gravitational pull of the central sun the disk commences to collapse. Typical dynamical timescales are of the order of the local Keplerian revolution period being shortest - around $1 \mathrm{yr}-$ for the innermost part of our model disk. The main evolutionary phases are the violent dynamical "switch-on" phase and the ensuing quiescent accretion phase within which the flow is developing asymptotically into a stationary state. The first phase lasts only a few revolution periods of its outermost part, that is, a few tens of years. Heat and pressure waves travel through the disk, shock waves transform kinetic energy into heat which is eventually radiated away. Radiative losses of the - due to our choice of the initial data - surplus amount of energy have an efficient damping effect on the disk's internal motions. After a few tens of revolution periods, that is, after several hundred years, a quasistationary flow pattern emerges and the disk luminosity is sustained just by the "slow" viscous energy dissipation. Evidently, the final outcome is exactly what can be referred to as a fully 2-D model of a preplanetary accretion disk.

In the following Sects. 5.1-5.3, we first discuss the role of the water-gas shift reaction for producing $\mathrm{CO}_{2}$ in our model nebula. How much $\mathrm{CO}_{2}$ can actually be produced has become an important question, since $\mathrm{CO}_{2}$ has recently been observed in the inner regions ( $\$ 10 \mathrm{AU}$ ) of protostellar disks (Lahuis et al. 2006). We then outline briefly the main results relating to the initial "switch-on" phase. Finally, we discuss the role of the advective material transport driven by large-scale circulations in the disk as compared to the diffusive transport and mixing processes that are expected to take place in turbulent media anyway.

\subsection{The water-gas shift reaction}

Among the various chemical reactions related to the basic chemistry of the molecules containing carbon, hydrogen, and oxygen (except hydrocarbons) we focused our attention, in the first instance, to the so-called water-gas shift reaction,

$\mathrm{CO}+\mathrm{H}_{2} \mathrm{O} \leftrightarrows \mathrm{CO}_{2}+\mathrm{H}_{2}$

comprising the forward and reverse reaction, reaction \#55 and \#54 in Table 4, respectively. If this reaction became effective in preplanetary nebulae in some way or another, it could serve as the most efficient source of carbon dioxide. It is well known 
Table 2. The initial data.

\begin{tabular}{lcll}
\hline \hline Quantity & Symbol & \multicolumn{1}{c}{ Value } & Dimension \\
\hline Central mass & $M_{\star}$ & 1 & $M_{\odot}$ \\
Disk mass & $M_{\text {disk }}$ & 0.01 & $M_{\odot}$ \\
Radial extension & $r$ & $0.8 \leq r \leq 5.8$ & $\mathrm{AU}$ \\
Vertical extension & $z$ & $-1.5 \leq z \leq 1.5$ & $\mathrm{AU}$ \\
Radial velocity & $u_{r}$ & 0 & $\mathrm{~km} \mathrm{~s}^{-1}$ \\
Vertical velocity & $u_{z}$ & 0 & $\mathrm{~km} \mathrm{~s}^{-1}$ \\
Azimuthal velocity & $u_{\phi}$ & $\sim 33 \ldots 12$ (Keplerian rotation) & $\mathrm{km} \mathrm{s}^{-1}$ \\
Density & $\rho$ & $1.91 \times 10^{-11}$ & $\mathrm{~g} \mathrm{~cm}^{-3}$ \\
Gas temperature & $T_{\text {kin }}$ & 20 & $\mathrm{~K}$ \\
Radiation temperature & $T_{\mathrm{rad}}$ & 20 & $\mathrm{~K}$ \\
Mean molecular weight & $\mu$ & 2.377 & - \\
Gas pressure & $P$ & $1.336 \times 10^{-2}$ & $\mathrm{dyn} \mathrm{cm}{ }^{-2}$ \\
Minimum ("vacuum") density & $\rho_{\mathrm{vac}}$ & $10^{-4} \rho=1.91 \times 10^{-15}$ & $\mathrm{~g} \mathrm{~cm}^{-3}$ \\
\hline Species & $\mathrm{Abundances} \mathrm{(concentrations)} \mathrm{per} \mathrm{weight}$ & \\
\hline Molecular hydrogen, $\mathrm{H}_{2}$ & $c_{\mathrm{H}_{2}}$ & 0.708933 & \\
Helium, He & $c_{\mathrm{He}}$ & 0.274615 & \\
Molecular nitrogen, $\mathrm{N}_{2}$ & $c_{\mathrm{N}_{2}}$ & 0.000920 & \\
Carbon monoxide, CO & $c_{\mathrm{CO}}$ & 0.006997 & \\
Water (ice mantle), $\mathrm{H}_{2} \mathrm{O}$ (solid) & $c_{\mathrm{H}_{2} \mathrm{O}}$ & 0.003020 & \\
"Dust" $\dagger$ & $c_{\text {dust }}$ & 0.005515 & \\
\hline & $\sum_{X} c_{X} \equiv 1$ & \\
\hline
\end{tabular}

${ }^{\dagger}$ Dust grains consisting mainly of forsterite $\left(\mathrm{Mg}_{2} \mathrm{SiO}_{4}\right)$ with an admixture of corundum $\left(\mathrm{Al}_{2} \mathrm{O}_{3}\right), \mathrm{Fe}$, $\mathrm{S}$, and Ca.

that, at a temperature around $1100 \mathrm{~K}$, the water-gas shift reaction is used to produce, via carbon combustion, a mixture of $\mathrm{H}_{2}$, $\mathrm{CO}$, and $\mathrm{CO}_{2}$, with a surplus of water vapor present, on a large technical scale. This is why the chemical equilibrium constant is of practical importance only in the high-temperatures range of 800-2000 K (see, e.g., Günther 1974; Kraus 2003); at lower temperatures the water-gas shift reaction is becoming inefficient for technical applications.

Above all, the equilibrium constant, $K_{p}$, of an arbitrary chemical reaction,

$s_{1}^{(\mathrm{r})} X_{1}+\ldots+s_{n}^{(\mathrm{r})} X_{n} \rightarrow s_{1}^{(\mathrm{p})} Y_{1}+\ldots+s_{m}^{(\mathrm{p})} Y_{m}$,

with $n$ reactants, $X_{1}, \ldots, X_{n}$, and $m$ products, $Y_{1}, \ldots, Y_{m}$, and the respective stoichiometric coefficients, $s_{1}^{(\mathrm{r})}, \ldots, s_{n}^{(\mathrm{r})}$ and $s_{1}^{(\mathrm{p})}, \ldots, s_{m}^{(\mathrm{p})}$, is available for any temperature by making use of the thermodynamic relation,

$\mathcal{R} T \cdot \ln \left(K_{p}\right)=-\sum_{i=1}^{n} s_{i}^{(\mathrm{r})} \Delta G_{X_{i}}+\sum_{j=1}^{m} s_{j}^{(\mathrm{p})} \Delta G_{Y_{j}}$.

The quantities, $\Delta G_{X_{i}}$ and $\Delta G_{Y_{j}}$, refer to the free enthalpy of formation of the species, $X_{i}$ and $Y_{j}$, respectively. For a huge variety of chemical compounds the necessary data to compute the $\Delta G_{X, Y}$ are compiled, e.g., in Chase Jr. (1998).

Whereas the equilibrium constant of the water-gas shift reaction can easily be calculated, there seems to be an almost complete lack of measurements and/or calculated numbers for the rate coefficients. Until recently, there was only one - meanwhile to be considered entirely obsolete - reference to the rate constant of the reaction, $\mathrm{H}_{2}+\mathrm{CO}_{2} \rightarrow \mathrm{H}_{2} \mathrm{O}+\mathrm{CO}$ (reaction \#54 in Table 4), in the UMIST data base (Le Teuff et al. 2000). The Arrhenius parameters that were given there are of category "C", i.e., the rate constant itself ought to be accurate within a factor of two in the temperature range of 268-300 K. However, based on quantum chemical investigations Talbi \& Herbst (2002) pointed out that the pure gas-phase reaction, $\mathrm{CO}_{2}+\mathrm{H}_{2} \rightarrow \mathrm{CO}+$ $\mathrm{H}_{2} \mathrm{O}$, “. . . possesses an extremely large potential energy barrier,
Table 3. Properties of the model sequences.

\begin{tabular}{ccc}
\hline \hline sequence & $\begin{array}{c}\text { Schmidt } \\
\text { number, } \mathcal{S}\end{array}$ & $\begin{array}{c}\text { water-gas shift reaction: } \\
\text { rate constants }\end{array}$ \\
\hline A & 1 & realistic (Talbi \& Herbst 2002) \\
B1 & 1 & obsolete (Le Teuff et al. 2000) \\
B2 & $\infty$ & obsolete (Le Teuff et al. 2000) \\
\hline
\end{tabular}

far in excess of the reaction endothermicity." (cf. reaction \#54 in Table 4).

As a consequence, the water-gas shift reaction in the pure gas-phase is ruled out as a main source of carbon dioxide in preplanetary nebulae. However, the presence of an appropriate catalytic agent, e.g., elementary iron (cf. Ford 1981), could raise the efficiency of the water-gas shift reaction substantially. In principle, micron-sized particles of pure iron are to exist in preplanetary nebulae, unless there is a major contamination by sulphuric compounds (e.g., $\mathrm{H}_{2} \mathrm{~S}$ ) so as to transform iron (Fe) gradually into troilite (FeS), which would again turn down the importance of the water-gas shift reaction.

With regard to the large uncertainties related to the watergas shift reaction, we conducted our simulations with the new as well as with the obsolete rate constants. Altogether, we have considered two distinct model categories, A and B, with characteristics as listed in Table 3. In the category A the most realistic rate constants according to Talbi \& Herbst (2002) are used; the two model sequences, B1 and B2, served as very first pilot studies and are devoted to explore the effects of diffusive mixing by choosing the Schmidt number, $\mathcal{S}=1$ and $\mathcal{S}=\infty$, respectively. Hence, concerning the water-gas shift reaction, in the model sequences of category $\mathrm{B}$, we have still used the (obsolete) UMIST rate constants as defined by (40) and (41) below.

In the sequel, we shall label quantities relating to the forward and reverse reaction by " $\rightarrow$ " and " $\leftarrow$ ", respectively. 
Table 4. The network for the gas-phase C-, H-, O-chemistry. The three Arrhenius-numbers, $A$ (in $\mathrm{cm}^{3} \mathrm{~s}^{-1}$ and $\mathrm{cm}^{6} \mathrm{~s}^{-1}$ for two- and three-particle reactions, respectively), $b$, and $T_{0}$ (in $\mathrm{K}$ ), represent the respective rate constant, $k(T)=A T^{b} \exp \left(-T_{0} / T\right)$, at the temperature, $T$. $Q_{\text {chem }}$ is the heat of formation (in $\mathrm{kJ} / \mathrm{mol}$ ).

\begin{tabular}{|c|c|c|c|c|c|c|c|c|}
\hline \# & \multicolumn{2}{|c|}{ chemical reaction } & $A$ & $b$ & $T_{0}$ & $Q_{\text {chem }}$ & $T$-range & source \\
\hline 1 & $\mathrm{H}+\mathrm{H}+\mathrm{H}$ & $\rightarrow \mathrm{H}_{2}+\mathrm{H}$ & $8.82 \mathrm{E}-33$ & 0.000 & 0 . & -433.52 & $50-5000 \mathrm{~K}$ & (3) \\
\hline 2 & $\mathrm{H}+\mathrm{H}_{2}$ & $\rightarrow \mathrm{H}+\mathrm{H}+\mathrm{H}$ & $4.50 \mathrm{E}-08$ & -0.100 & 52500. & 433.52 & $600-5000 \mathrm{~K}$ & (3) \\
\hline 3 & $\mathrm{H}+\mathrm{H}+\mathrm{H}_{2}$ & $\rightarrow \mathrm{H}_{2}+\mathrm{H}_{2}$ & $2.70 \mathrm{E}-31$ & -0.600 & 0 . & -433.52 & $100-5000 \mathrm{~K}$ & (2) \\
\hline 4 & $\mathrm{H}_{2}+\mathrm{H}_{2}$ & $\rightarrow \mathrm{H}+\mathrm{H}+\mathrm{H}_{2}$ & $1.50 \mathrm{E}-09$ & 0.000 & 48350. & 433.52 & $2500-8000 \mathrm{~K}$ & (2) \\
\hline 5 & $\mathrm{H}_{2}+\mathrm{OH}$ & $\rightarrow \mathrm{H}_{2} \mathrm{O}+\mathrm{H}$ & $1.70 \mathrm{E}-16$ & 1.600 & 1660. & -62.82 & $300-2500 \mathrm{~K}$ & (2) \\
\hline 6 & $\mathrm{H}+\mathrm{H}_{2} \mathrm{O}$ & $\rightarrow \mathrm{OH}+\mathrm{H}_{2}$ & $7.50 \mathrm{E}-16$ & 1.600 & 9270. & 62.82 & $300-2500 \mathrm{~K}$ & (2) \\
\hline 7 & $\mathrm{H}+\mathrm{OH}$ & $\rightarrow \mathrm{H}_{2}+\mathrm{O}$ & $8.10 \mathrm{E}-21$ & 2.800 & 1950. & -7.81 & $300-2500 \mathrm{~K}$ & (13) \\
\hline 8 & $\mathrm{O}+\mathrm{H}_{2}$ & $\rightarrow \mathrm{OH}+\mathrm{H}$ & $8.50 \mathrm{E}-20$ & 2.670 & 3160. & 7.81 & $300-2500 \mathrm{~K}$ & (2) \\
\hline 9 & $\mathrm{OH}+\mathrm{OH}$ & $\rightarrow \mathrm{H}_{2} \mathrm{O}+\mathrm{O}$ & $2.50 \mathrm{E}-15$ & 1.140 & 50. & -70.63 & $250-2500 \mathrm{~K}$ & (2) \\
\hline 10 & $\mathrm{O}+\mathrm{H}_{2} \mathrm{O}$ & $\rightarrow \mathrm{OH}+\mathrm{OH}$ & $8.20 \mathrm{E}-14$ & 0.950 & 8571. & 70.63 & $250-2400 \mathrm{~K}$ & (8) \\
\hline 11 & $\mathrm{O}+\mathrm{OH}$ & $\rightarrow \mathrm{O}_{2}+\mathrm{H}$ & $2.40 \mathrm{E}-11$ & 0.000 & 353. & -70.16 & $1000-2000 \mathrm{~K}$ & (2) \\
\hline 12 & $\mathrm{H}+\mathrm{O}_{2}$ & $\rightarrow \mathrm{OH}+\mathrm{O}$ & $1.62 \mathrm{E}-10$ & 0.000 & 7470. & 70.16 & $300-5000 \mathrm{~K}$ & (2) \\
\hline 13 & $\mathrm{H}+\mathrm{HO}_{2}$ & $\rightarrow \mathrm{H}_{2}+\mathrm{O}_{2}$ & $7.10 \mathrm{E}-11$ & 0.000 & 710. & -220.09 & $300-1000 \mathrm{~K}$ & (2) \\
\hline 14 & $\mathrm{H}_{2}+\mathrm{O}_{2}$ & $\rightarrow \mathrm{HO}_{2}+\mathrm{H}$ & $2.41 \mathrm{E}-10$ & 0.000 & 28505 . & 220.09 & $300-2500 \mathrm{~K}$ & (13) \\
\hline 15 & $\mathrm{H}_{2}+\mathrm{O}_{2}$ & $\rightarrow \mathrm{OH}+\mathrm{OH}$ & $3.16 \mathrm{E}-10$ & 0.000 & 21900. & 77.97 & $800-1250 \mathrm{~K}$ & (1) \\
\hline 16 & $\mathrm{OH}+\mathrm{OH}$ & $\rightarrow \mathrm{H}_{2}+\mathrm{O}_{2}$ & $8.90 \mathrm{E}-12$ & 0.000 & 12520. & -77.97 & & (cbr) to $\# 15$ \\
\hline 17 & $\mathrm{O}+\mathrm{HO}_{2}$ & $\rightarrow \mathrm{OH}+\mathrm{O}_{2}$ & $5.30 \mathrm{E}-11$ & 0.000 & 0 . & -212.28 & $300-1000 \mathrm{~K}$ & (2) \\
\hline 18 & $\mathrm{OH}+\mathrm{O}_{2}$ & $\rightarrow \mathrm{O}+\mathrm{HO}_{2}$ & $3.70 \mathrm{E}-11$ & 0.000 & 26500. & 212.28 & $300-2500 \mathrm{~K}$ & (13) \\
\hline 19 & $\mathrm{H}+\mathrm{HO}_{2}$ & $\rightarrow \mathrm{OH}+\mathrm{OH}$ & $2.80 \mathrm{E}-11$ & 0.000 & 440. & -142.12 & $300-1000 \mathrm{~K}$ & (2) \\
\hline 20 & $\mathrm{OH}+\mathrm{OH}$ & $\rightarrow \mathrm{H}+\mathrm{HO}_{2}$ & $5.81 \mathrm{E}-15$ & 0.000 & 17530. & 142.12 & & (cbr) to $\# 19$ \\
\hline 21 & $\mathrm{H}+\mathrm{HO}_{2}$ & $\rightarrow \mathrm{H}_{2} \mathrm{O}+\mathrm{O}$ & $5.00 \mathrm{E}-11$ & 0.000 & 866. & -212.74 & $300-1000 \mathrm{~K}$ & (2) \\
\hline 22 & $\mathrm{H}_{2} \mathrm{O}+\mathrm{O}$ & $\rightarrow \mathrm{H}+\mathrm{HO}_{2}$ & $1.13 \mathrm{E}-13$ & 0.000 & 26450. & 212.74 & & (cbr) to $\# 21$ \\
\hline 23 & $\mathrm{H}+\mathrm{H}_{2} \mathrm{O}_{2}$ & $\rightarrow \mathrm{H}_{2}+\mathrm{HO}_{2}$ & $2.80 \mathrm{E}-12$ & 0.000 & 1890. & -79.80 & $300-1000 \mathrm{~K}$ & (2) \\
\hline 24 & $\mathrm{H}_{2}+\mathrm{HO}_{2}$ & $\rightarrow \mathrm{H}_{2} \mathrm{O}_{2}+\mathrm{H}$ & $5.00 \mathrm{E}-11$ & 0.000 & 13100. & 79.80 & $300-2500 \mathrm{~K}$ & (13) \\
\hline 25 & $\mathrm{OH}+\mathrm{HO}_{2}$ & $\rightarrow \mathrm{H}_{2} \mathrm{O}+\mathrm{O}_{2}$ & $2.91 \mathrm{E}-11$ & 0.000 & 200. & -282.90 & $300-2500 \mathrm{~K}$ & (15) \\
\hline 26 & $\mathrm{H}_{2} \mathrm{O}+\mathrm{O}_{2}$ & $\rightarrow \mathrm{OH}+\mathrm{HO}_{2}$ & 7.72E-12 & 0.000 & 37300. & 282.90 & $300-1000 \mathrm{~K}$ & (10) \\
\hline 27 & $\mathrm{H}+\mathrm{H}_{2} \mathrm{O}_{2}$ & $\rightarrow \mathrm{OH}+\mathrm{H}_{2} \mathrm{O}$ & $1.70 \mathrm{E}-11$ & 0.000 & 1800. & -284.72 & $300-1000 \mathrm{~K}$ & (2) \\
\hline 28 & $\mathrm{OH}+\mathrm{H}_{2} \mathrm{O}$ & $\rightarrow \mathrm{H}+\mathrm{H}_{2} \mathrm{O}_{2}$ & $2.85 \mathrm{E}-12$ & 0.000 & 36040. & 284.72 & & (cbr) to \#27 \\
\hline 29 & $\mathrm{O}+\mathrm{H}_{2} \mathrm{O}_{2}$ & $\rightarrow \mathrm{OH}+\mathrm{HO}_{2}$ & $1.10 \mathrm{E}-12$ & 0.000 & 2000. & -71.99 & $300-500 \mathrm{~K}$ & (2) \\
\hline 30 & $\mathrm{OH}+\mathrm{HO}_{2}$ & $\rightarrow \mathrm{O}+\mathrm{H}_{2} \mathrm{O}_{2}$ & $2.90 \mathrm{E}-13$ & 0.000 & 10658. & 71.99 & & (cbr) to \#29 \\
\hline 31 & $\mathrm{HO}_{2}+\mathrm{HO}_{2}$ & $\rightarrow \mathrm{H}_{2} \mathrm{O}_{2}+\mathrm{O}_{2}$ & $3.11 \mathrm{E}-12$ & 0.000 & 775. & -140.29 & $550-1250 \mathrm{~K}$ & (2) \\
\hline 32 & $\mathrm{O}_{2}+\mathrm{H}_{2} \mathrm{O}_{2}$ & $\rightarrow \mathrm{HO}_{2}+\mathrm{HO}_{2}$ & $9.00 \mathrm{E}-11$ & 0.000 & 20000. & 140.29 & $300-2500 \mathrm{~K}$ & (13) \\
\hline 33 & $\mathrm{OH}+\mathrm{H}_{2} \mathrm{O}_{2}$ & $\rightarrow \mathrm{H}_{2} \mathrm{O}+\mathrm{HO}_{2}$ & $1.30 \mathrm{E}-11$ & 0.000 & 670. & -142.62 & $300-1000 \mathrm{~K}$ & (2) \\
\hline 34 & $\mathrm{HO}_{2}+\mathrm{H}_{2} \mathrm{O}$ & $\rightarrow \mathrm{H}_{2} \mathrm{O}_{2}+\mathrm{OH}$ & $4.60 \mathrm{E}-11$ & 0.000 & 16500. & 142.62 & $300-1000 \mathrm{~K}$ & (9) \\
\hline 35 & $\mathrm{H}+\mathrm{H}_{2} \mathrm{O}$ & $\rightarrow \mathrm{OH}+\mathrm{H}+\mathrm{H}$ & $5.80 \mathrm{E}-09$ & 0.000 & 52900. & 501.29 & $2000-6000 \mathrm{~K}$ & (2) \\
\hline 36 & $\mathrm{OH}+\mathrm{H}+\mathrm{H}_{2}$ & $\rightarrow \mathrm{H}_{2} \mathrm{O}+\mathrm{H}_{2}$ & $2.30 \mathrm{E}-26$ & -2.000 & 0 . & -501.29 & $300-3000 \mathrm{~K}$ & (2) \\
\hline 37 & $\mathrm{H}_{2}+\mathrm{H}_{2} \mathrm{O}$ & $\rightarrow \mathrm{OH}+\mathrm{H}+\mathrm{H}_{2}$ & $5.80 \mathrm{E}-09$ & 0.000 & 52900. & 501.29 & $2000-6000 \mathrm{~K}$ & (2) \\
\hline 38 & $\mathrm{OH}+\mathrm{H}+\mathrm{H}_{2}$ & $\rightarrow \mathrm{H}_{2} \mathrm{O}+\mathrm{H}_{2}$ & $6.10 \mathrm{E}-26$ & -2.000 & 0 . & -501.29 & $300-3000 \mathrm{~K}$ & (2) \\
\hline 39 & $\mathrm{H}+\mathrm{OH}$ & $\rightarrow \mathrm{O}+\mathrm{H}+\mathrm{H}$ & $4.00 \mathrm{E}-09$ & 0.000 & 50000. & 430.66 & $300-2500 \mathrm{~K}$ & (13) \\
\hline 40 & $\mathrm{O}+\mathrm{H}+\mathrm{H}$ & $\rightarrow \mathrm{OH}+\mathrm{H}$ & $1.30 \mathrm{E}-29$ & -1.000 & 0. & -430.66 & $300-2500 \mathrm{~K}$ & (13) \\
\hline 41 & $\mathrm{H}_{2}+\mathrm{OH}$ & $\rightarrow \mathrm{O}+\mathrm{H}+\mathrm{H}_{2}$ & $4.00 \mathrm{E}-09$ & 0.000 & 50000. & 430.66 & $300-2500 \mathrm{~K}$ & (13) \\
\hline 42 & $\mathrm{O}+\mathrm{H}+\mathrm{H}_{2}$ & $\rightarrow \mathrm{OH}+\mathrm{H}_{2}$ & $1.30 \mathrm{E}-29$ & -1.000 & 0. & -430.66 & $300-2500 \mathrm{~K}$ & (13) \\
\hline 43 & $\mathrm{H}_{2}+\mathrm{H}_{2} \mathrm{O}_{2}$ & $\rightarrow \mathrm{OH}+\mathrm{OH}+\mathrm{H}_{2}$ & 2.01E-07 & 0.000 & 22900. & 216.56 & $700-1500 \mathrm{~K}$ & (2) \\
\hline 44 & $\mathrm{OH}+\mathrm{OH}+\mathrm{H}_{2}$ & $\rightarrow \mathrm{H}_{2} \mathrm{O}_{2}+\mathrm{H}_{2}$ & $8.00 \mathrm{E}-31$ & -0.780 & 0. & -216.56 & $250-1400 \mathrm{~K}$ & (2) \\
\hline 45 & $\mathrm{H}+\mathrm{O}_{2}$ & $\rightarrow \mathrm{O}+\mathrm{O}+\mathrm{H}$ & $3.00 \mathrm{E}-06$ & -1.000 & 59400. & 500.82 & $300-2500 \mathrm{~K}$ & (13) \\
\hline 46 & $\mathrm{H}+\mathrm{O}+\mathrm{O}$ & $\rightarrow \mathrm{O}_{2}+\mathrm{H}$ & $5.21 \mathrm{E}-35$ & 0.000 & -900 & -500.82 & $200-4000 \mathrm{~K}$ & (13) \\
\hline 47 & $\mathrm{H}_{2}+\mathrm{O}_{2}$ & $\rightarrow \mathrm{O}+\mathrm{O}+\mathrm{H}_{2}$ & $3.00 \mathrm{E}-06$ & -1.000 & 59400. & 500.82 & $300-2500 \mathrm{~K}$ & (13) \\
\hline 48 & $\mathrm{H}_{2}+\mathrm{O}+\mathrm{O}$ & $\rightarrow \mathrm{O}_{2}+\mathrm{H}_{2}$ & $5.21 \mathrm{E}-35$ & 0.000 & -900 . & -500.82 & $200-4000 \mathrm{~K}$ & (13) \\
\hline 49 & $\mathrm{H}_{2}+\mathrm{HO}_{2}$ & $\rightarrow \mathrm{O}_{2}+\mathrm{H}+\mathrm{H}_{2}$ & $2.00 \mathrm{E}-05$ & -1.180 & 25400. & 215.91 & $200-2200 \mathrm{~K}$ & (13) \\
\hline 50 & $\mathrm{H}_{2}+\mathrm{H}+\mathrm{O}_{2}$ & $\rightarrow \mathrm{HO}_{2}+\mathrm{H}_{2}$ & $5.80 \mathrm{E}-30$ & -0.800 & 0 . & -215.91 & $300-2000 \mathrm{~K}$ & (2) \\
\hline 51 & $\mathrm{H}+\mathrm{O}$ & $\rightarrow \mathrm{OH}+h v$ & $9.90 \mathrm{E}-19$ & -0.380 & 0 . & -430.66 & & (12) \\
\hline
\end{tabular}

\subsubsection{The equilibrium constant}

We are now going to discuss in more detail how to derive the missing rate constant, $k_{\rightarrow}$, in the Arrhenius form if both $k_{\leftarrow}$ and the equilibrium constant, $K_{p}$, are known. By definition of chemical equilibrium, $K_{p}$ is expressed as the ratio of the rate constants, for instance, $K_{p}:=k_{\leftarrow} / k_{\rightarrow}$. From this relation the (formal) solution, $k_{\rightarrow}=k_{\leftarrow} / K_{p}$, follows immediately. Obviously, this kind of reasoning is valid for any conjugated pair of chemical reactions. Moreover, if two of the three quantities, $k_{\leftarrow}, k_{\rightarrow}, K_{p}$, are represented in the Arrhenius form, so must be the third one in order to maintain consistency.

In Kraus (2003) the equilibrium constant, $K_{p}$, is approximated by a sixth order polynomial in the temperature range $500-1600{ }^{\circ} \mathrm{C}$, i.e., roughly $800-1900 \mathrm{~K}$ :

$K_{p}(T)=\sum_{i=0}^{6} q_{i} \cdot\left(\frac{T}{1{ }^{\circ} \mathrm{C}}\right)^{i}$, 
Table 4. continued.

\begin{tabular}{|c|c|c|c|c|c|c|c|c|}
\hline \# & \multicolumn{2}{|c|}{ chemical reaction } & $A$ & $b$ & $T_{0}$ & $Q_{\text {chem }}$ & $T$-range & source \\
\hline 52 & $\mathrm{OH}+\mathrm{CO}$ & $\rightarrow \mathrm{H}+\mathrm{CO}_{2}$ & $1.05 \mathrm{E}-17$ & 1.500 & -250 . & -103.95 & $300-2000 \mathrm{~K}$ & $(2)$ \\
\hline 53 & $\mathrm{H}+\mathrm{CO}_{2}$ & $\rightarrow \mathrm{OH}+\mathrm{CO}$ & $2.50 \mathrm{E}-10$ & 0.000 & 13300. & 103.95 & $300-2500 \mathrm{~K}$ & (13) \\
\hline 54 & $\mathrm{H}_{2}+\mathrm{CO}_{2}$ & $\rightarrow \mathrm{H}_{2} \mathrm{O}+\mathrm{CO}$ & $1.05 \mathrm{E}-15$ & 1.500 & 56900. & 41.14 & $1000-2000 \mathrm{~K}$ & (14), cf. Sect. 5.1 \\
\hline 55 & $\mathrm{H}_{2} \mathrm{O}+\mathrm{CO}$ & $\rightarrow \mathrm{H}_{2}+\mathrm{CO}_{2}$ & $1.92 \mathrm{E}-21$ & 2.687 & 51561. & -41.14 & & (cbr) to $\# 54$ \\
\hline 56 & $\mathrm{O}_{2}+\mathrm{CO}$ & $\rightarrow \mathrm{CO}_{2}+\mathrm{O}$ & $4.20 \mathrm{E}-12$ & 0.000 & 24000. & -33.79 & $300-2500 \mathrm{~K}$ & (13) \\
\hline 57 & $\mathrm{O}+\mathrm{CO}_{2}$ & $\rightarrow \mathrm{CO}+\mathrm{O}_{2}$ & $2.80 \mathrm{E}-11$ & 0.000 & 26500. & 33.79 & $300-2500 \mathrm{~K}$ & (13) \\
\hline 58 & $\mathrm{CO}+\mathrm{HO}_{2}$ & $\rightarrow \mathrm{CO}_{2}+\mathrm{OH}$ & $2.50 \mathrm{E}-10$ & 0.000 & 11900. & -246.07 & $300-2500 \mathrm{~K}$ & (13) \\
\hline 59 & $\mathrm{CO}_{2}+\mathrm{OH}$ & $\rightarrow \mathrm{CO}+\mathrm{HO}_{2}$ & $1.18 \mathrm{E}-11$ & 0.000 & 41500 . & 246.07 & & (cbr) to \#58 \\
\hline 60 & $\mathrm{H}+\mathrm{HCO}$ & $\rightarrow \mathrm{CO}+\mathrm{H}_{2}$ & $1.50 \mathrm{E}-10$ & 0.000 & 0 . & -372.05 & $300-2500 \mathrm{~K}$ & (2) \\
\hline 61 & $\mathrm{CO}+\mathrm{H}_{2}$ & $\rightarrow \mathrm{H}+\mathrm{HCO}$ & $6.77 \mathrm{E}-10$ & 0.000 & 44750. & 372.05 & & (cbr) to \#60 \\
\hline 62 & $\mathrm{O}+\mathrm{HCO}$ & $\rightarrow \mathrm{OH}+\mathrm{CO}$ & $5.00 \mathrm{E}-11$ & 0.000 & 0 . & -364.24 & $300-2000 \mathrm{~K}$ & (2) \\
\hline 63 & $\mathrm{OH}+\mathrm{CO}$ & $\rightarrow \mathrm{O}+\mathrm{HCO}$ & $1.02 \mathrm{E}-10$ & 0.000 & 43810. & 364.24 & & (cbr) to \#62 \\
\hline 64 & $\mathrm{O}+\mathrm{HCO}$ & $\rightarrow \mathrm{CO}_{2}+\mathrm{H}$ & $5.00 \mathrm{E}-11$ & 0.000 & 0 . & -486.19 & $300-2000 \mathrm{~K}$ & (2) \\
\hline 65 & $\mathrm{CO}_{2}+\mathrm{H}$ & $\rightarrow \mathrm{O}+\mathrm{HCO}$ & $2.70 \mathrm{E}-07$ & 0.000 & 58480. & 486.19 & & (cbr) to \#64 \\
\hline 66 & $\mathrm{OH}+\mathrm{HCO}$ & $\rightarrow \mathrm{H}_{2} \mathrm{O}+\mathrm{CO}$ & $1.70 \mathrm{E}-10$ & 0.000 & 0 . & -434.87 & $300-2000 \mathrm{~K}$ & (2) \\
\hline 67 & $\mathrm{H}_{2} \mathrm{O}+\mathrm{CO}$ & $\rightarrow \mathrm{OH}+\mathrm{HCO}$ & $3.76 \mathrm{E}-09$ & 0.000 & 52300. & 434.87 & & (cbr) to \#66 \\
\hline 68 & $\mathrm{H}+\mathrm{H}_{2} \mathrm{CO}$ & $\rightarrow \mathrm{HCO}+\mathrm{H}_{2}$ & $2.10 \mathrm{E}-16$ & 1.620 & 1090. & -58.58 & $300-1700 \mathrm{~K}$ & (2) \\
\hline 69 & $\mathrm{HCO}+\mathrm{H}_{2}$ & $\rightarrow \mathrm{H}+\mathrm{H}_{2} \mathrm{CO}$ & $3.00 \mathrm{E}-19$ & 2.000 & 8970. & 58.58 & $300-2500 \mathrm{~K}$ & (13) \\
\hline 70 & $\mathrm{O}+\mathrm{H}_{2} \mathrm{CO}$ & $\rightarrow \mathrm{HCO}+\mathrm{OH}$ & $6.90 \mathrm{E}-13$ & 0.570 & 1390. & -50.77 & $250-2200 \mathrm{~K}$ & (2) \\
\hline 71 & $\mathrm{OH}+\mathrm{HCO}$ & $\rightarrow \mathrm{O}+\mathrm{H}_{2} \mathrm{CO}$ & $1.67 \mathrm{E}-14$ & 0.570 & 7500. & 50.77 & & (cbr) to \#71 \\
\hline 72 & $\mathrm{OH}+\mathrm{H}_{2} \mathrm{CO}$ & $\rightarrow \mathrm{H}_{2} \mathrm{O}+\mathrm{HCO}$ & $5.70 \mathrm{E}-15$ & 1.180 & 225. & -121.40 & $300-3000 \mathrm{~K}$ & (2) \\
\hline 73 & $\mathrm{H}_{2} \mathrm{O}+\mathrm{HCO}$ & $\rightarrow \mathrm{OH}+\mathrm{H}_{2} \mathrm{CO}$ & $3.90 \mathrm{E}-16$ & 1.350 & 1310. & 121.40 & $300-2500 \mathrm{~K}$ & (13) \\
\hline 74 & $\mathrm{O}_{2}+\mathrm{H}_{2} \mathrm{CO}$ & $\rightarrow \mathrm{HO}_{2}+\mathrm{HCO}$ & $1.00 \mathrm{E}-10$ & 0.000 & 20460. & 161.51 & $700-1000 \mathrm{~K}$ & (2) \\
\hline 75 & $\mathrm{HO}_{2}+\mathrm{HCO}$ & $\rightarrow \mathrm{O}_{2}+\mathrm{H}_{2} \mathrm{CO}$ & 7.27E-10 & 0.000 & 1030. & -161.51 & & (cbr) to \#74 \\
\hline 76 & $\mathrm{HCO}+\mathrm{HCO}$ & $\rightarrow \mathrm{H}_{2} \mathrm{CO}+\mathrm{CO}$ & $5.00 \mathrm{E}-11$ & 0.000 & 0 . & -313.47 & $300-2500 \mathrm{~K}$ & (2) \\
\hline 77 & $\mathrm{H}_{2} \mathrm{CO}+\mathrm{CO}$ & $\rightarrow \mathrm{HCO}+\mathrm{HCO}$ & 4.22E-09 & 0.000 & 37700. & 313.47 & & (cbr) to \#76 \\
\hline 78 & $\mathrm{H}_{2} \mathrm{O}_{2}+\mathrm{HCO}$ & $\rightarrow \mathrm{HO}_{2}+\mathrm{H}_{2} \mathrm{CO}$ & $1.69 \mathrm{E}-13$ & 0.000 & 3490. & -21.22 & $300-2500 \mathrm{~K}$ & (13) \\
\hline 79 & $\mathrm{HO}_{2}+\mathrm{H}_{2} \mathrm{CO}$ & $\rightarrow \mathrm{H}_{2} \mathrm{O}_{2}+\mathrm{HCO}$ & $5.00 \mathrm{E}-12$ & 0.000 & 6580. & 21.22 & $600-1000 \mathrm{~K}$ & (2) \\
\hline 80 & $\mathrm{CO}+\mathrm{H}$ & $\rightarrow \mathrm{C}+\mathrm{O}+\mathrm{H}$ & $6.00 \mathrm{E}-09$ & 0.000 & 129000. & 1076.34 & & (11) \\
\hline 81 & $\mathrm{C}+\mathrm{O}+\mathrm{H}$ & $\rightarrow \mathrm{CO}+\mathrm{H}$ & $2.00 \mathrm{E}-34$ & 0.000 & 0 . & -1076.34 & $8000 \mathrm{~K}$ & (6) \\
\hline 82 & $\mathrm{H}+\mathrm{CO}_{2}$ & $\rightarrow \mathrm{CO}+\mathrm{O}+\mathrm{H}$ & $6.46 \mathrm{E}-10$ & 0.000 & 53900. & 532.14 & $3600-6500 \mathrm{~K}$ & (7) \\
\hline 83 & $\mathrm{H}+\mathrm{CO}+\mathrm{O}$ & $\rightarrow \mathrm{CO}_{2}+\mathrm{H}$ & $1.70 \mathrm{E}-33$ & 0.000 & 1510. & -532.14 & $300-2500 \mathrm{~K}$ & (13) \\
\hline 84 & $\mathrm{H}_{2}+\mathrm{CO}_{2}$ & $\rightarrow \mathrm{CO}+\mathrm{O}+\mathrm{H}_{2}$ & $6.46 \mathrm{E}-10$ & 0.000 & 53900. & 532.14 & $3600-6500 \mathrm{~K}$ & (7) \\
\hline 85 & $\mathrm{H}_{2}+\mathrm{CO}+\mathrm{O}$ & $\rightarrow \mathrm{CO}_{2}+\mathrm{H}_{2}$ & $1.70 \mathrm{E}-33$ & 0.000 & 1510. & -532.14 & $300-2500 \mathrm{~K}$ & (13) \\
\hline 86 & $\mathrm{HCO}+\mathrm{H}_{2}$ & $\rightarrow \mathrm{CO}+\mathrm{H}+\mathrm{H}_{2}$ & $2.61 \mathrm{E}-10$ & 0.000 & 7930. & 63.94 & $600-2500 \mathrm{~K}$ & (2) \\
\hline 87 & $\mathrm{CO}+\mathrm{H}+\mathrm{H}_{2}$ & $\rightarrow \mathrm{HCO}+\mathrm{H}_{2}$ & $5.30 \mathrm{E}-34$ & 0.000 & 370. & -63.94 & $300-2500 \mathrm{~K}$ & (2) \\
\hline 88 & $\mathrm{H}_{2}+\mathrm{H}_{2} \mathrm{CO}$ & $\rightarrow \mathrm{HCO}+\mathrm{H}+\mathrm{H}_{2}$ & $2.00 \mathrm{E}+17$ & -6.900 & 48600 . & 377.41 & $1000-3000 \mathrm{~K}$ & (13) \\
\hline 89 & $\mathrm{H}_{2}+\mathrm{HCO}+\mathrm{H}$ & $\mathrm{H} \rightarrow \mathrm{H}_{2} \mathrm{CO}+\mathrm{H}_{2}$ & $7.31 \mathrm{E}-24$ & -2.570 & 215. & -377.41 & $1560-2270 \mathrm{~K}$ & $(5)$ \\
\hline 90 & $\mathrm{C}+\mathrm{O}$ & $\rightarrow \mathrm{CO}+h v$ & $6.09 \mathrm{E}-20$ & 0.732 & 0 . & -1076.34 & & (4) \\
\hline
\end{tabular}

Sources of data: (1) Azatyan et al. (1975); (2) Baulch et al. (1992); (3) Cohen \& Westberg (1983); (4) Dalgarno et al. (1990); (5) Eiteneer et al. (1998); (6) Fairbairn (1969); (7) Kiefer (1974); (8) Lifschitz \& Michael (1991); (9) Lloyd (1974); (10) Mayer \& Schieler (1968); (11) Mitchell (1984); (12) Le Teuff et al. (2000); (13) Tsang \& Hampson (1986); (14) Talbi \& Herbst (2002); (15) Warnatz, private communication; (cbr) computed back-reaction.

Three-particle reactions are in most cases estimated from corresponding reactions with noble gases or $\mathrm{N}_{2}$ as third species.

with the coefficients

$$
\begin{array}{ll}
q_{0}=4.8095 \times 10^{-1}, & q_{1}=-2.9970 \times 10^{-3}, \\
q_{2}=6.1170 \times 10^{-6}, & q_{3}=-3.8770 \times 10^{-9} \\
q_{4}=3.9220 \times 10^{-12}, & q_{5}=-2.3900 \times 10^{-15}, \\
q_{6}=4.7059 \times 10^{-19} . &
\end{array}
$$

Furthermore, by applying Eq. (37) to the water-gas shift reaction, we obtained a list of $K_{p}$-values from the relevant data compiled in Chase Jr. (1998) in the temperature range between $0 \mathrm{~K}$ and $6000 \mathrm{~K}$.

Both representations of $K_{p}$ can easily be transformed by a standard best-fit procedure into the desired Arrhenius form for $K_{p}\left(\right.$ in $\left.\mathrm{cm}^{3} \mathrm{~s}^{-1}\right)$ :

$K_{p}=5.484 \times 10^{5} T^{-1.187} \exp (-5339 / T)$.

The result is displayed in Fig. 2. In our applications we restricted ourselves to temperatures below $2000 \mathrm{~K}$. In the high-temperature regime (right panel), the Arrhenius-fit (full line) is practically identical with both the polynomial representation (dash-dot line) and the numbers derived from the Chase Jr. (1998) data (dashed line). Likewise, the lower left panel of Fig. 2 shows the variation of $K_{p}$ for low temperatures. Again, the Arrhenius approximation (full line) matches remarkably well the calculated Chase Jr. (1998) values (dashed line).

Realistic rate constants. According to Talbi \& Herbst (2002) the rate constant, $k_{\leftarrow}$, of the water-gas shift reaction, $\mathrm{CO}_{2}+$ $\mathrm{H}_{2} \rightarrow \mathrm{CO}+\mathrm{H}_{2} \mathrm{O}$ (reaction \#54), is several orders of magnitude lower than the one given in the UMIST data base (Le Teuff et al. 2000, see Eq. (41) below).

Obsolete rate constants. Since there is an almost complete lack of both experimental and theoretical data for the watergas shift reaction at low temperatures, we are free to choose a 

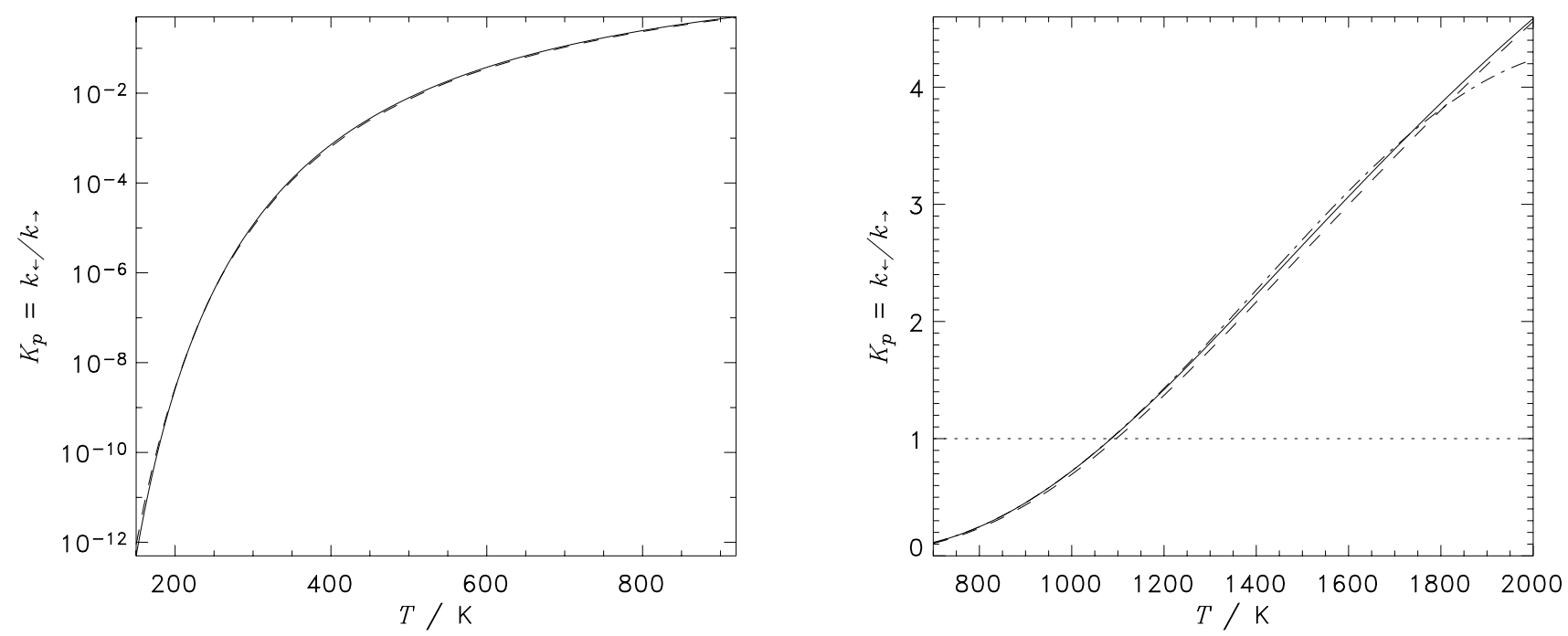

Fig. 2. The water-gas shift reaction, $\mathrm{CO}+\mathrm{H}_{2} \mathrm{O} \leftrightarrows \mathrm{CO}_{2}+\mathrm{H}_{2}$ : Arrhenius form of the equilibrium constant, $K_{p}:=k_{\leftarrow} / k_{\rightarrow}$, for lower $($ left panel) and higher (right panel) temperatures. Dash-dot line: polynomial representation according to Kraus (2003) (cf. Eq. (38)). Seemingly, for higher temperatures above 1800-1900 K the deviations are increasing rapidly. Dashed lines: computed equilibrium constant from the thermodynamical data for the species $\mathrm{CO}, \mathrm{CO}_{2}, \mathrm{H}_{2} \mathrm{O}, \mathrm{H}_{2}$ in Chase Jr. (1998).

practically arbitrary pair of rate constants. For example, based on the UMIST data (Le Teuff et al. 2000) the respective rate constants read

$k_{\rightarrow}=3.10 \times 10^{-21} T^{1.687} \exp (-2211 / T)$,
$k_{\leftarrow}=1.70 \times 10^{-15} T^{0.500} \exp (-7550 / T)$.

From the known (UMIST) Arrhenius parameters of the reverse water-gas shift reaction, $k_{\leftarrow}: \mathrm{CO}_{2}+\mathrm{H}_{2} \rightarrow \mathrm{CO}+\mathrm{H}_{2} \mathrm{O}$, the parameters of the forward reaction, $k_{\rightarrow}$ : $\mathrm{CO}+\mathrm{H}_{2} \mathrm{O} \rightarrow \mathrm{CO}_{2}+\mathrm{H}_{2}$ are tailored so as to be consistent with the equilibrium constant, $K_{p}:=k_{\leftarrow} / k_{\rightarrow}$ (cf. Fig. 2). The (obsolete) rate constants, (40) and (41), are used only for exploring the importance of the diffusive material transport in the vertical direction. Comparative studies of this kind, by varying the Schmidt number, $\mathcal{S}$, are carried out in the model sequences B1 and B2.

\subsection{The dynamical "switch-on" phase}

Since we start out with a homogeneous and isothermal disk-like configuration, vertical pressure gradients have not yet built up to compensate the vertical gravitational pull the central protosun exerts on the disk material. As a consequence, the initial configuration commences to collapse essentially in the z-direction, while the radial direction is less affected. This is due to the fact that the initial disk is a Keplerian one (cf. Table 2) and is therefore radially sustained by centrifugal forces.

Figure 3 displays the contour lines of several important physical quantities according to their distribution in the $(r, z)$-plane. It represents a snapshot of the non-stationary collapse-like "switch-on" phase of our model disk after one revolution period as measured at a radial distance of 1 AU. As expected, the highest compression occurs in the innermost regions of the disk near the equatorial plane. This happens also at the inner boundary, which is an artifact of our adopted "almost-rigid-wall" condition: the mass flow across the inner border of the disk ceases with vanishing viscosity. Though highly artificial, mastering such a complex dynamical situation is, nevertheless, again a firm test for the robustness of the code. In the very early phases (within about the first $0.2 \mathrm{yr}$ ) Mach numbers of above 40 were present, declining to about 11 after $1 \mathrm{yr}$ (cf. Fig. 3, lowest left panel) and to 4.5 after $12 \mathrm{yr}$ which is the revolution time of the outermost disk layers ( $r \lesssim 5.8 \mathrm{AU}$ ). In other words, the flow exhibits strong shock fronts almost from the beginning. However, they become weaker and eventually disappear when after several dynamical time scales, i.e., several tens of years, a smooth viscosity-driven accretion flow remains, which is on the way to asymptotically approach a stationary state.

The temperatures in the maximum compression zones rise well above the silicate dust sublimation temperature, thus creating a deep opacity gap and, accordingly, a noticeable difference between the kinetic and the radiation temperature. The hot gas cannot cool fast enough because the dust, as the principal absorber, does not exist anymore, while the radiation energy quickly diffuses away (cf. the panels of Fig. 3 showing the distribution of the opacity, radiation temperature, and kinetic temperature, respectively). A heat wave originating from the hot interior and a pressure wave propagate radially toward the outer regions of the disk and the "snow line" is continually shifted to larger radii.

Along with the rather violent dynamical and thermal evolution of our model disk the various gas-phase reactions in the chemical network comprising 90 reactions are activated in the "warm" zones. This is illustrated by Fig. 4 for the "most realistic" case A. It shows the distribution of all reactive gaseous species (except those not exceeding a minimum concentration of $10^{-15}$ ) that we have taken into consideration, again at an age of $1 \mathrm{yr}$. Carbon dioxide $\left(\mathrm{CO}_{2}\right)$ is produced to some extent in the hottest regions of the disk.

\subsection{Asymptotic, quasi-stationary accretion phase}

In the following we discuss results of the numerical experiments pertaining to the mechanical/thermal structure of the disk and to the abundance and spatial distribution of the various gas species at a evolutionary time of $300 \mathrm{yr}$. We focus our attention on the relation between advective and diffusive mixing by varying the Schmidt number. 



Fig. 3. Sequence A. Snapshot of the mechanical and thermal structure of the model disk after $1 \mathrm{yr}$, i.e., one revolution of the disk around the central protosun at a radial distance of 1 AU. Depicted are the contour lines of the different physical quantities. The left uppermost and the second right panel from above show the distribution of the density and the radiation temperature, and the arrow fields represent the velocity field and the radiative flux (in erg $\mathrm{cm}^{-2} \mathrm{~s}^{-1}$ ), respectively. The scales are indicated by the single arrow in the lower right corner of the respective panels. The opacity gap relates to the high temperature region where the dust particles evaporate (panels in the second row from below); it is also visible in the depression of the contours of equal vertical optical depths, $\tau_{z}$. The "surface" of the disk defined by $\tau_{z}=1$ is indicated by the heavy contour line (third left panel from below). The "snow line" coincides exactly with the head of the heat wave and the accompanying pressure wave travelling through the disk in the outward direction (right panels). The bottom row shows the distribution of the Mach numbers, Ma, and the sound speed, $c_{\mathrm{s}}$, in the left and right panel, respectively. The tick marks perpendicular to the Ma contour lines point toward the "downhill" direction.

\subsubsection{Mechanical and thermal disk structure}

In all our simulations so far carried out, we have been dealing with just one "mean" dust species having ice mantles at low temperatures, $T \lesssim 150 \mathrm{~K}$, which determines the absorption properties of the disk material. The much lower gas opacities that depend on the chemistry become important only at higher temperatures, when the refractory grains evaporate and the opacity gap opens (cf. Fig. 3, second row of panels from below). Since we have not yet taken into account effects of detailed gas-solid-phase chemistry (heteromolecular evaporation and condensation) and mineralogical metamorphosis such as annealing, which both determine the optical properties of the dust grains and, hence, the opacity of the disk material to a large extent, the overall mechanical and thermal properties of our model disks are essentially the same for all three model sequences, A, B1, B2 (cf. Table 3). 
A

CHO gas-phase chemistry, time: $1.0 \mathrm{yr}$
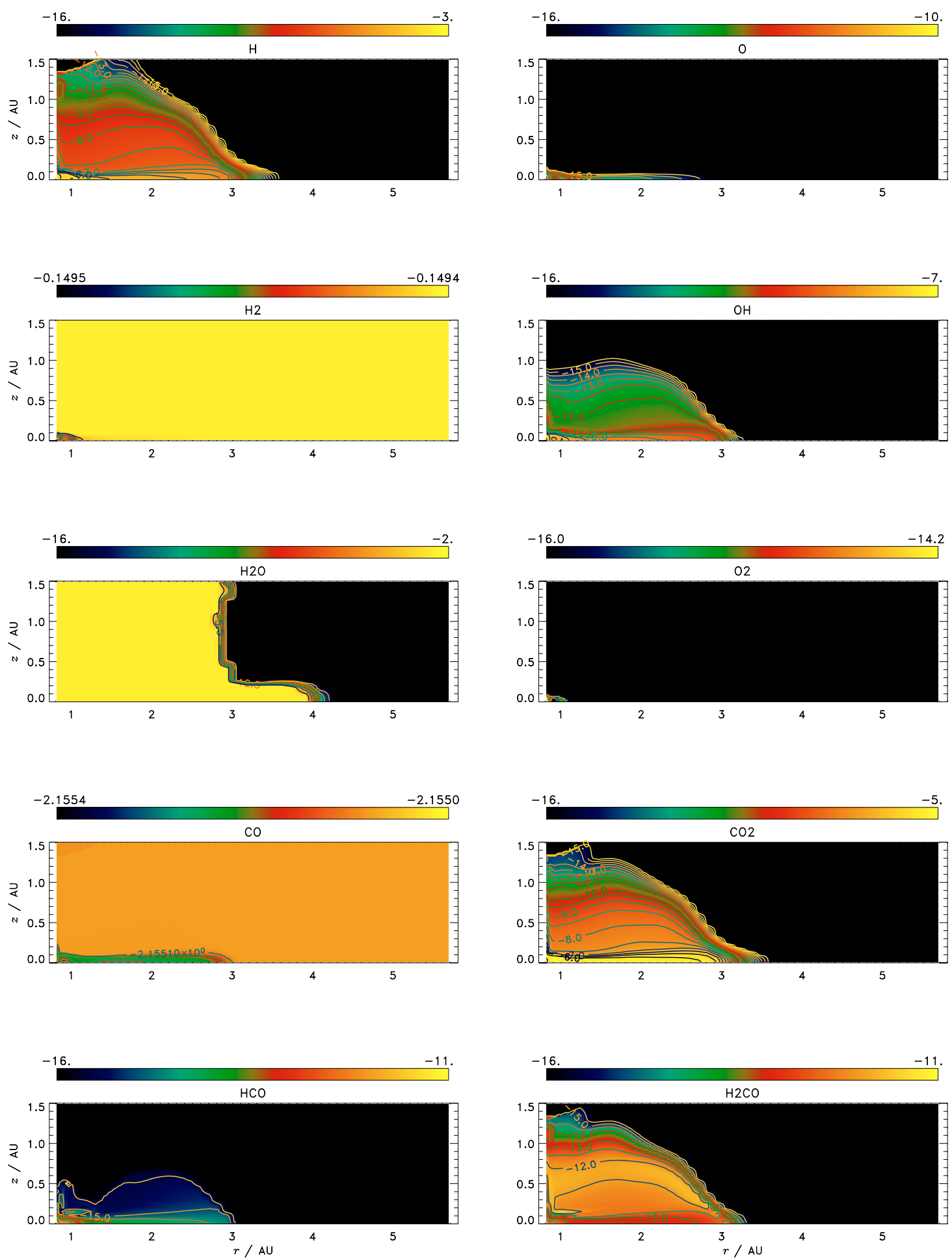

Fig. 4. Sequence A. Distribution of the concentration, $c$, that is, the relative abundance (per weight) of the gaseous species after 1 yr, i.e., after one revolution of the disk around the central protosun at a radial distance of $1 \mathrm{AU}$. The title line of the panels contains the respective chemical formula of the species whose concentration contour lines are shown. The color bar indicates the range in between the concentrations, $c$, vary (logarithmic) over the $(r, z)$-plane. The lower $\operatorname{limit}$ of $\log (c)=-16$ means zero abundance. In the panel showing the distribution of water vapor $\left(\mathrm{H}_{2} \mathrm{O}\right.$, first panel in the third row) the snow line (cf. Fig. 3) is again visible as a distinct feature. Because of the extremely low abundance of the species, $\mathrm{HO}_{2}, \mathrm{H}_{2} \mathrm{O}_{2}$, and (gaseous) $\mathrm{C}$, their respective concentrations, $c$ (altogether $<10^{-15}$ ), are not shown.

Figure 5 shows the distribution of the physical quantities after $300 \mathrm{yr}$ or about 20 revolution periods of the outermost parts of our model disk that is gradually evolving into a stationary accretion state. Quasi-hydrostatic equilibrium in the vertical direction has already been established, the velocities within the disk are extremely subsonic. The Mach numbers 

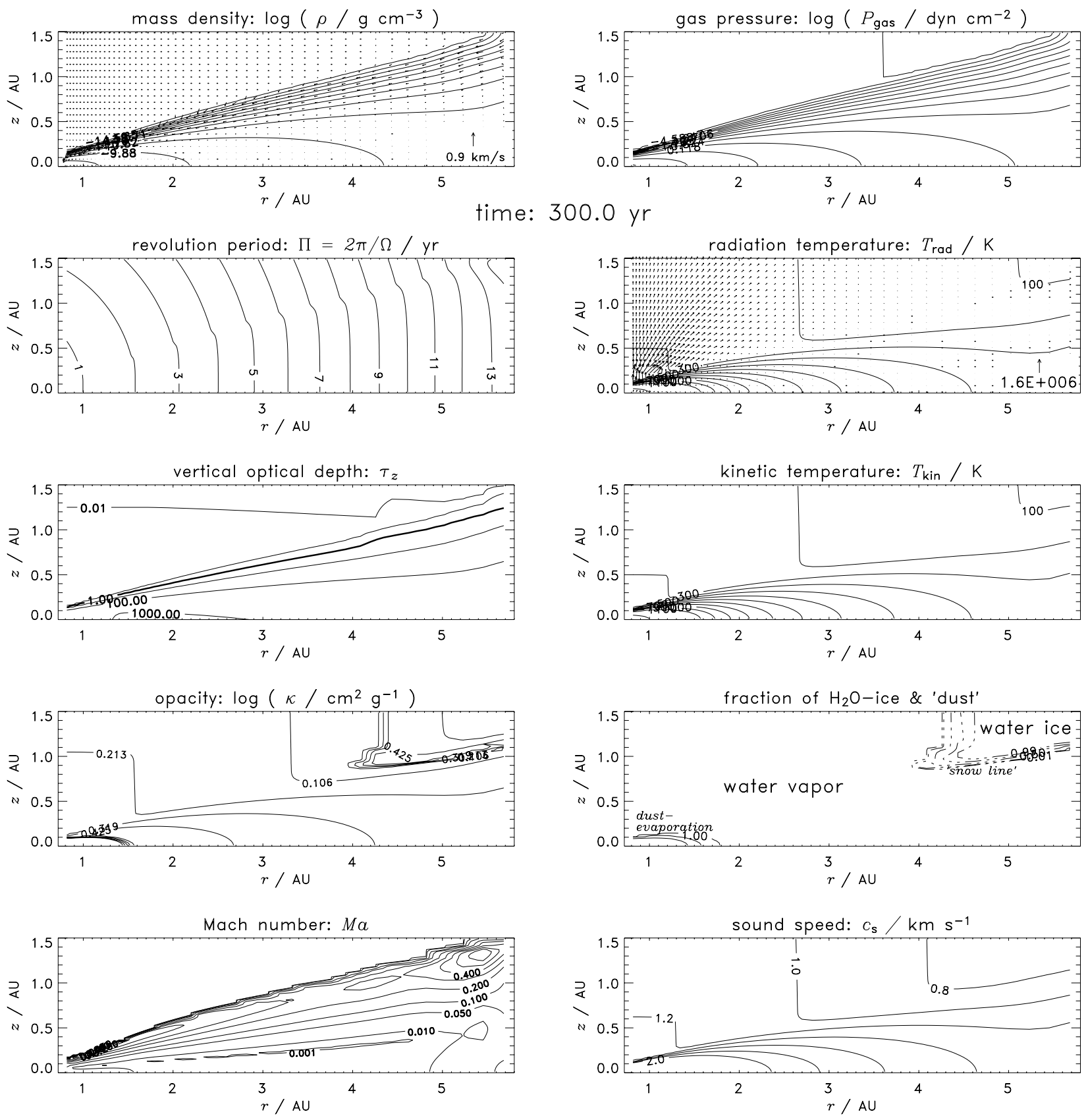

Fig. 5. Sequence A. The same drawings of the physical quantities as in Fig. 3, but at the end of the simulation. Somewhat less than 500000 timesteps were necessary to cover the entire evolutionary period of $300 \mathrm{yr}$. The radiating "surface" at unity vertical optical depth, $\tau_{z}=1$, is emphasized by the heavy contour line in the left third panel from above. Note the extremely low Mach numbers, Ma, particularly in the region of 1-2 scale heights $(z / r \simeq 0.1 \ldots 0.25)$ "above" the equator $(z=0)$; the intermediate Mach numbers in the upper right corner of the panel are a result of the somewhat artificial inflow boundary condition: the mass elements entering at high altitudes find themselves off mechanical equilibrium and gain velocity under the surplus gravitational pull.

reached within the scale height of the disk, that is, within its "half-thickness", $h / r \simeq 0.12$ (cf. Fig. 7b), are considerably smaller than 0.01. The fastest motions occur in the innermost regions of the disk, slightly above its "surface", within the narrow transition zone where the density rises above the fixed minimum density, $\rho_{\text {vac }}=1.91 \times 10^{-15} \mathrm{~g} \mathrm{~cm}^{-3}$ (cf. Sect. 4.4). The second left panel from above displays the distribution of the revolution periods over the entire $(r, z)$-plane. The sub-domain containing the disk proper is clearly visible. There the action of the viscous forces drives the disk to establish a rotation law with angular velocities being constant on cylindrical surfaces, that is, $\partial \Omega / \partial z \rightarrow 0$, which is indicated by the contour lines of equal revolution period, $\Pi:=2 \pi / \Omega \equiv 2 \pi r / u_{\phi}$, in Fig. 5 , second left panel from above.

The maximum optical depth of the disk in the vertical direction,

$\tau_{z}:=-\int_{z_{\max }}^{z} \kappa \rho \mathrm{d} z$

counted from the upper boundary of our integration domain $\left(z=z_{\max }:=1.5 \mathrm{AU}\right)$ down to the disk's "equator" $(z=0)$, is slightly greater than $10^{3}$ (cf. Fig. 5, third left panel from above). In the innermost parts of the disk, around $1 \mathrm{AU}$ in the radial 

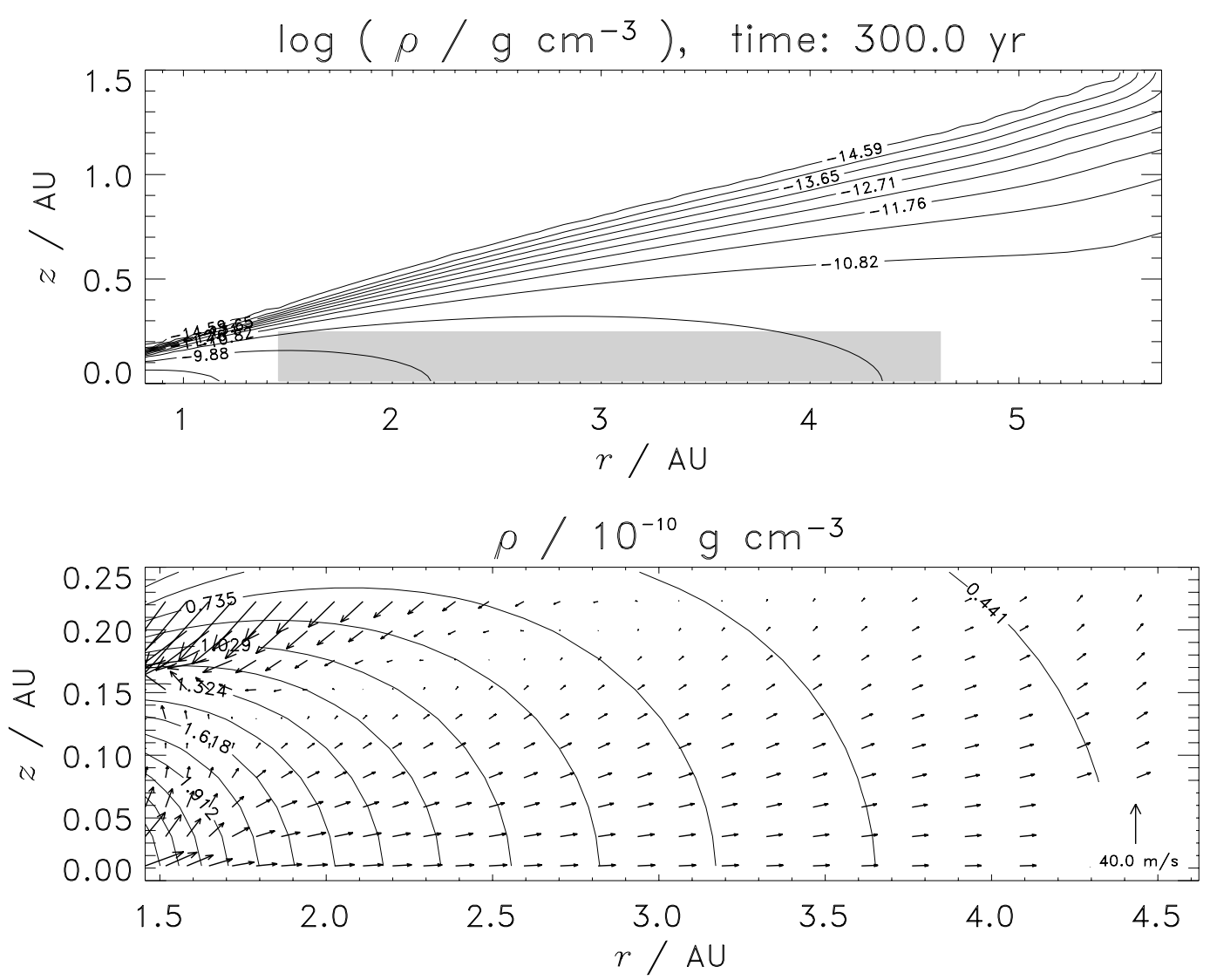

Fig. 6. Sequence A. Density contours and the velocity field after 300 years. Upper panel: overall view of the density distribution. Lower panel: the magnified region as indicated by the grey-shaded rectangle in the upper panel. The outward motion of about $10-20 \mathrm{~m} \mathrm{~s}^{-1}$ near the equatorial plane is clearly visible.

direction and extending to about $0.1 \mathrm{AU}$ in the vertical direction the temperatures remain sufficiently high $(\gtrsim 1300 \mathrm{~K})$ so that the dust particles evaporate and leave behind again an opacity gap. On the cool ( $\$ 150 \mathrm{~K})$ edge at large radial distances (around $5 \mathrm{AU})$ and high altitudes $(\gtrsim 0.8 \ldots 0.9 \mathrm{AU})$ water vapor condenses out on the dust particles to form ice-coated grains, in this way defining the "snow line" as depicted in Fig. 5, second right panel from below.

As is illustrated in Fig. 3, second and third right panel from above, during the highly non-equilibrium "switch-on phase" the (equivalent) radiation temperature, $T_{\mathrm{rad}}$, and the kinetic temperature, $T_{\text {kin }}:=T$, may differ locally, e.g., due to drastically reduced optical depths (formation of opacity gaps as a result of dust evaporation) or to compression waves and, though not particularly exemplified here, to shock fronts exhibiting extended radiative cooling zones if travelling in optically thin regions. However, at our reference epoch of $300 \mathrm{yr}$, besides the mechanical equilibrium an almost perfect thermal equilibrium has been established: $T_{\text {rad }}=T_{\text {kin }}$ throughout the disk, as is documented in Fig. 5, second and third right panel from above. The opacity gap has retracted to the hottest innermost part of the disk (cf. the "opacity mountain" depicted in Fig. 9).

\subsubsection{Circulation currents}

A result of major importance is displayed in Fig. 6. It corresponds to the early qualitative finding that Kippenhahn \& Thomas (1982) derived in an extensive analytical investigation on the compatibility of thermal and hydrostatic equilibrium in thin radiative accretion disks. Within the framework of Keplerian $\alpha$-disks, the authors distinguish between two different cases dependent on $\alpha$ being smaller or greater than the relative halfthickness, $h / r$, of the disk, respectively. Owing to energy conservation, mechanical and thermal equilibria in the strict sense cannot exist simultaneously. As a compensation for this discrepancy, the mass elements are put in motion to form a pronounced circulation pattern within the disk. If now $\alpha<h / r$ (Case I according to Kippenhahn \& Thomas 1982) or $\alpha>h / r$ (Case II) the circulation is thermally or dynamically driven, respectively. Dissipation of energy by turbulent friction keeps the engine running.

Later, Urpin (1984) pointed out that the actual flow pattern in (quasi-stationary) 2-D accretion disks is decisively more complicated than the simple radial accretion flow which is inherent to any one-zone or (1+1)-D model. Other investigations (e.g., Keller 2003; Keller \& Gail 2004; Siemiginowska 1988; Kley et al. 1993; Różyczka et al. 1994; Regev \& Gitelman 2002) have confirmed that the axially symmetric accretion flow exhibits a huge circulation pattern. Near the equatorial plane, well below one scale height of the disk where the mass density is highest, a slowly moving flow in the outward direction develops. The much faster, inwardly directed back flow occurs at larger altitudes, extending up to the disk's "surface" which is situated, by definition, at the vertical optical depth, $\tau_{z}=1$. There, the dissipated energy is radiated away and the density decreases rapidly with increasing vertical distance, $z$.

According to the relation (19) between the $\alpha$ - and $\beta$-parameter and the result of our simulations pertaining to the half-thickness, $h / r$, of the disk we may state: for the inner part, 

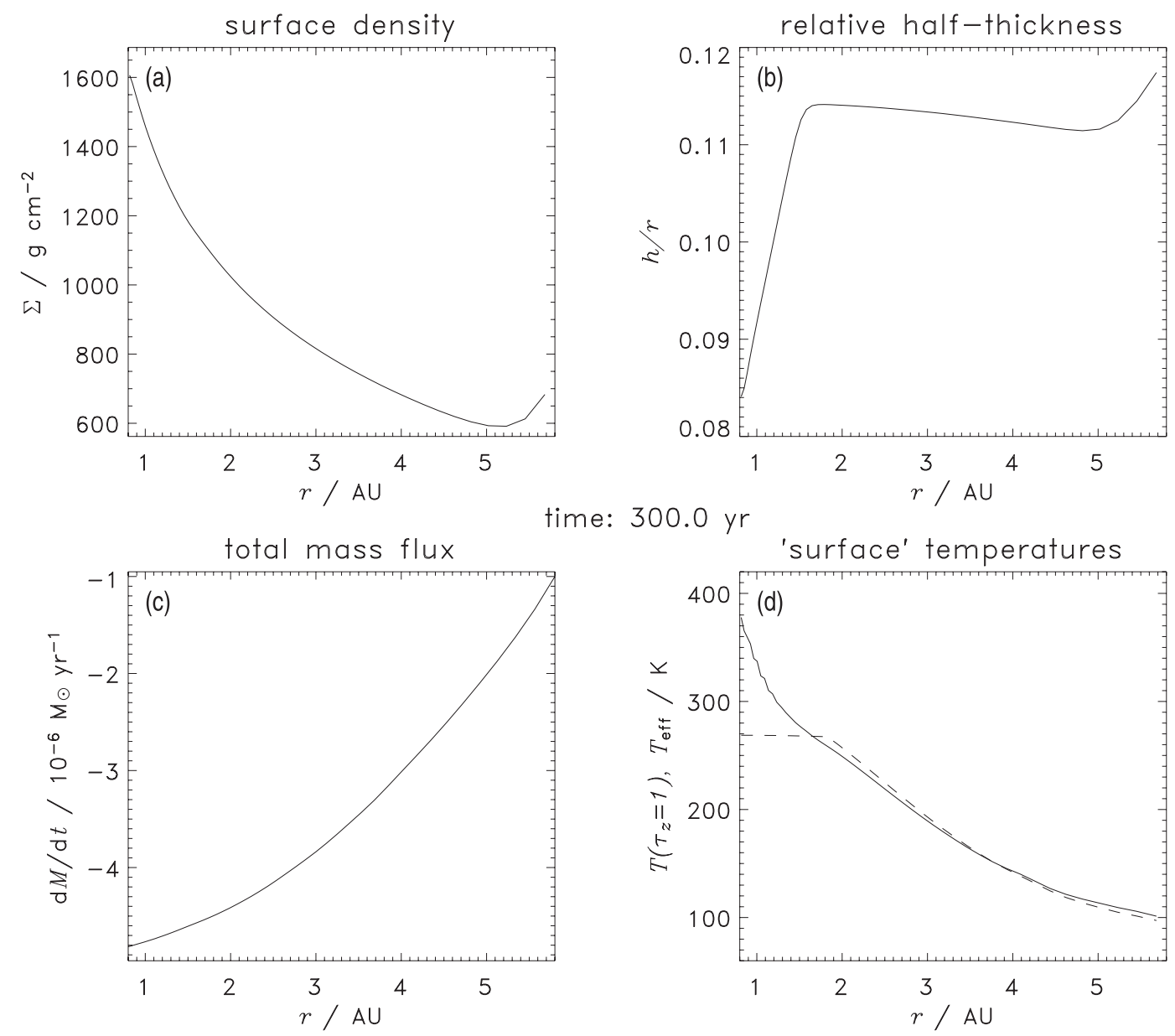

Fig. 7. Sequence A. Mechanical and thermal structure of the disk after $300 \mathrm{yr}$ (cf. Fig. 5). Radial variation of physical quantities integrated over the disk's vertical dimension. a) Surface density, $\Sigma$ (cf. Eq. (49)). The increase toward the outer boundary is a consequence of the particularly chosen mass-inflow boundary condition. b) Half-thickness, $h / r$, of the disk (cf. Eq. (50)). Corresponding to the behavior of the surface density (see panel a), $h / r$ increases in the outermost parts of the disk, again being an artifact of the outer boundary condition. The relatively steep decrease of $h / r$ toward the inner boundary is due to a combination of the inner boundary condition and a lack of numerical resolution. c) Net mass accretion rate, $\dot{M} \equiv \mathrm{d} M / \mathrm{d} t$ (cf. Eq. (51)). The strictly stationary state for which the condition, $\dot{M}=$ const, holds is not yet established: there is still more mass leaking out of the disk through the inner boundary than is entering the disk at the outer boundary. d) "Surface" temperatures. Full line: gas temperature, $T\left(\tau_{z}=1\right)$, at the optical depth, $\tau_{z}=1$, counted from the "upper" boundary $\left(z=z_{\max }:=1.5 \mathrm{AU}\right)$ down to the disk's equatorial plane $(z=0)$ in the vertical direction. Dashed line: effective temperature, $T_{\text {eff }}$ (cf. Eq. (52)). The conspicuous deviation from $T\left(\tau_{z}=1\right)$ in the innermost parts is to be attributed to both poor numerical resolution and the flux-limiting procedure for the Eddington approximation.

$2 \lesssim r / \mathrm{AU} \lesssim 5$, sufficiently far off the inner and outer "rim" we find $h / r \approx$ const. $\simeq 0.115$ (cf. $7 \mathrm{~b}$ ) and, together with $\beta=10^{-3}$ being our canonical choice, $\alpha=0.076<h / r$. This inequality refers to Case I in the classification scheme of Kippenhahn \& Thomas (1982), i.e., our circulation depicted in Fig. 6 is essentially thermally driven. Note that $h / r \equiv 0.1$ indicates the transition from Case I to Case II (dynamically driven circulation).

By integrating the mass flux over the vertical $(z-)$ coordinate, it turns out that there is a net radial mass influx, $\dot{M}$, throughout the disk. The typical velocities in the back flow are 1-2 orders of magnitude larger than those of the equatorial outward motion ranging in between $10-20 \mathrm{~m} \mathrm{~s}^{-1}$. The expected fact that, in addition to the remarkable large-scale circulation flow, there is a net accretion rate is depicted in Fig. 7c. Strict stationarity would demand $\dot{M}=\dot{M}_{\mathrm{ob}}=-10^{-6} M_{\odot} \mathrm{yr}^{-1}$ at every radial distance, $r$, according to our inflow boundary condition (cf. Sect. 4.4).

The radial variation of the net mass flux, in particular the difference between the fluxes at the outer and inner radial boundary, $\dot{M}_{\mathrm{ob}}$ and $\dot{M}_{\mathrm{ib}}$, respectively, is a measure of how closely stationarity has already been established. At our reference epoch of $300 \mathrm{yr}$ about five times as much mass is leaving the inner boundary as enters the disk at its outer boundary. A long-term test calculation has shown that after another $1300 \mathrm{yr}$ the ratio of the mass fluxes, $\dot{M}_{\mathrm{ib}} / \dot{M}_{\mathrm{ob}}$, would decrease from the initial number of five to about three. It is thus obvious that our model disk is asymptotically approaching a stationary state.

A sensitive indicator of how the model disk approaches the stationary state is the evolution of the total disk luminosity versus the total dissipation rate, $\dot{Q}_{\text {vis,tot }}$, due to turbulent friction, which is strongly enhanced by shock waves forming during the dynamical "switch-on" phase. As is displayed in Fig. 10, $\dot{Q}_{\text {vis,tot }}$ is a smooth function of time throughout (full line); by contrast, the total luminosity, $L_{\text {disk }}$, of the disk exhibits oscillations of rather large amplitudes (dashed line). This behavior mirrors the huge variations of the opacity in the inner disk regions where (instantaneous) sublimation and recondensation processes of the dust grains take place. Hence, small temperature variations can cause large changes in the opacity, which leads to largely varying radiative fluxes. After one revolution time of the disk as a whole ( $\approx 10 \mathrm{yr}$ ) the short-period variations of the 
A

CHO gas-phase chemistry, time: $300.0 \mathrm{yr}$
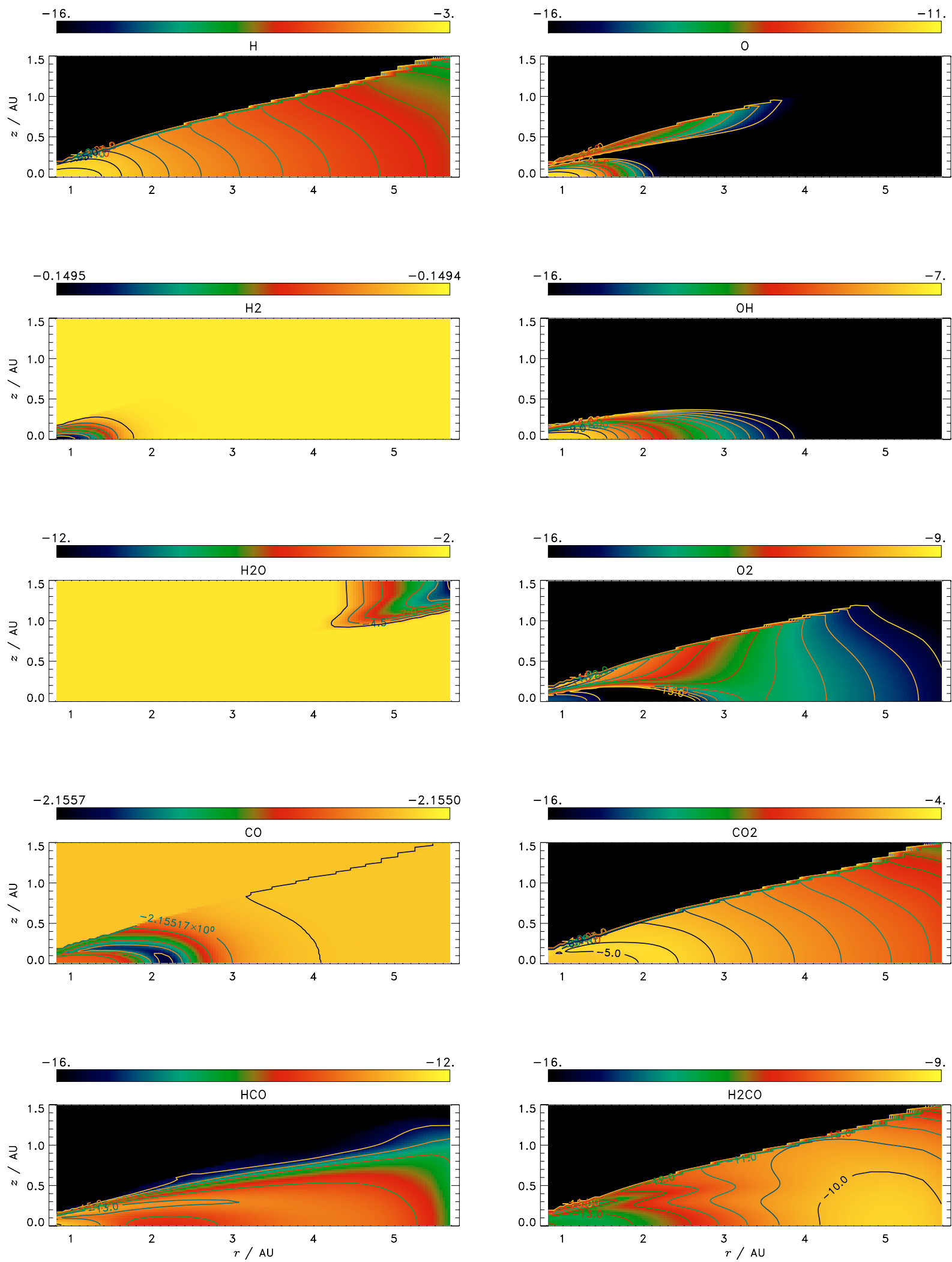

Fig. 8. Sequence A. The same drawings of the distribution of the chemical species as in Fig. 4, but at the end of the simulation, after 300 yr.

luminosity cease. During this "final" period, a slightly growing fraction $\left(L_{\text {disk }} / \dot{Q}_{\text {vis,tot }} \gtrsim 0.7\right)$ of the entire dissipated energy is radiated away; the remaining $30 \%$ partly keep the large-scale circulation (cf. Fig. 6) running, partly drive the enhanced mass outflow $\left(4.8 \ldots 3.8 \times 10^{-6} M_{\odot} / \mathrm{yr}\right.$ in between 300 and $\left.900 \mathrm{yr}\right)$ at the inner "rim" of the disk. 




Fig. 9. Sequence A. The "opacity mountain" associated with the disk model at an age of $300 \mathrm{yr}$. The inner gap and the outer enhancement of the opacity, $\kappa$, are caused by evaporation of the refractory dust component (silicates) and by condensation of water vapor to form ice-coated dust particles, respectively.



Fig. 10. Evolution of the total disk luminosity, and the total rate of energy dissipated by viscous processes (turbulence, shocks) in units of solar luminosity, $L_{\odot}$, as calculated for a three times more extended time span of about $900 \mathrm{yr}$ (as compared to our standard reference epoch of 300 yr). Full line: total energy dissipation rate, $\dot{Q}_{\text {vis,tot. Dashed line: }}$ disk luminosity, $L_{\mathrm{disk}}$. Dotted line: disk luminosity for strictly stationary 1 -D disk models according to Eq. (43) with mass flux $\dot{M}=10^{-6} M_{\odot} / \mathrm{yr}$.

The horizontal dotted line in Fig. 10 illustrates the disk luminosity according to a strictly stationary 1-D model. The respective expression is worked out, e.g., in Frank et al. (2002) and reads:

$$
\begin{aligned}
L_{\mathrm{disk}}\left(r_{\mathrm{ib}}, r_{\mathrm{ob}}\right)= & \frac{3 G M_{\star} \dot{M}}{2}\left[\frac{1}{r_{\mathrm{ib}}}\left(1-\frac{2}{3} \sqrt{\frac{R_{\star}}{r_{\mathrm{ib}}}}\right)\right. \\
& \left.-\frac{1}{r_{\mathrm{ob}}}\left(1-\frac{2}{3} \sqrt{\frac{R_{\star}}{r_{\mathrm{ob}}}}\right)\right],
\end{aligned}
$$

where $M_{\star}, R_{\star} \mathrm{cm}$ are the central star's mass and radius and $r_{\mathrm{ib}}, r_{\mathrm{ob}}$ the inner- and outermost radius of the disk, respectively; $G$ denotes the gravitational constant. For our disk model, we adopted the following set of numbers:

$$
\begin{array}{ll}
\dot{M}=10^{-6} M_{\odot} / \mathrm{yr} & \\
M_{\star}=1 M_{\odot}, & R_{\star}=R_{\odot} \simeq 7 \times 10^{10} \mathrm{~cm} \\
r_{\mathrm{ib}}=0.8 \mathrm{AU}, & r_{\mathrm{ob}}=5.8 \mathrm{AU} .
\end{array}
$$

Inserting these numbers into Eq. (43) yields a total disk luminosity, $L_{\text {disk }}\left(r_{\mathrm{ib}}, r_{\mathrm{ob}}\right)=0.22 L_{\odot}$.

\subsubsection{Mixing processes}

Such large-scale circulations are an efficient means to transport advectively material that has been processed in the warm inner regions of the disk into its cool outer parts. The time, $t_{\mathrm{adv}}$, it takes to transport a mass element over a radial distance, $\Delta r$, given a flow velocity, $u_{r}$, only slightly dependent on the radial distance, $r$, is easily estimated to be simply

$t_{\mathrm{adv}} \simeq \frac{\Delta r}{u_{r}}$

For our model disk with a maximum $\Delta r \simeq 5 \mathrm{AU}$ and an equatorial outward flow, $u_{r} \simeq 15 \mathrm{~m} \mathrm{~s}^{-1}$ (cf. Fig. 6), the corresponding $t_{\mathrm{adv}} \simeq 1500 \mathrm{yr}$. Accordingly, within our reference time span of $300 \mathrm{yr}$ a mass element near the disk's equator has travelled only a distance of somewhat less $1 \mathrm{AU}$. As an - admittedly risky - extrapolation of our numerical findings, by inserting an appropriate number, $\Delta r$, for the typical spatial extension of real preplanetary disks, e.g., $\Delta r=100 \mathrm{AU}$, we end up with a characteristic time scale, $t_{\mathrm{adv}} \simeq 30000 \mathrm{yr}$. This is an astounding short timescale, but even if $t_{\text {adv }}$ were to be 1-2 orders of magnitude larger, advective mass transport would play a significant role in the evolution of the preplanetary nebulae, provided the large-scale circulation pattern also evolves within, and is maintained for, the whole disk extension over such long periods of time.

It is interesting to compare the "advective" timescale, $t_{\mathrm{adv}}$, with the diffusive mixing timescale, $t_{\text {diff }}$, which is defined by

$t_{\mathrm{diff}}:=\frac{\Delta r^{2}}{D}$

with $D$ being the diffusion coefficient. According to the $\beta$-prescription (17) for the (turbulent) kinematic viscosity coefficient, $v_{\text {turb }}$, and the definition (3) of the Schmidt number, $\mathcal{S}$, we can express the diffusion coefficient in the form $D=\mathcal{S}^{-1} \beta \cdot r^{2} \Omega$. Hence, for Keplerian disks the proportionality $D \propto \sqrt{r}$ holds. The diffusive mixing timescale, $t_{\text {diff }}$, defined above in Eq. (45) can now be rewritten in the form

$t_{\mathrm{diff}}=\frac{\Pi}{2 \pi \beta} \cdot \mathcal{S} \cdot\left(\frac{\Delta r}{r}\right)^{2}$,

where $\Pi=\Pi\left(r ; M_{\star}\right)$ is the Keplerian rotation period of a mass element at the radial distance, $r$, and for a central mass, $M_{\star}$. Now $\Pi(r) \propto M_{\star}^{-0.5} r^{1.5}$ (Kepler's third law) and, hence, $t_{\text {diff }} \propto$ $M_{\star}^{-0.5} r^{1.5}(\Delta r / r)^{2}$, whereas $t_{\mathrm{adv}} \propto r(\Delta r / r)$ with the assumption that the velocity, $u_{r}$, in Eq. (44) is approximately constant. Since radial transport and mixing will become, by definition, effective if $\Delta r / r \simeq 1$, there exists a distinguished radial distance, $r_{\text {mix }}$, beyond which mass elements near the disk's equator are predominantly transported by advection. Obviously, $r_{\text {mix }}$ results from the condition that the advective and the diffusive mixing time scales be equal. In fact, if the equality $t_{\mathrm{adv}}=t_{\mathrm{diff}}$ is to hold, we find

$\frac{r_{\mathrm{mix}}}{1 \mathrm{AU}}=\frac{4}{\mathcal{S}^{2}}\left(\frac{\beta}{10^{-3}}\right)^{2}\left(\frac{u_{r}}{15 \mathrm{~m} \mathrm{~s}^{-1}}\right)^{-2}\left(\frac{M_{\star}}{1 M_{\odot}}\right)^{-1}$. 
An immediate consequence of the timescale estimates given above is that for our model disks with Schmidt number, $\mathcal{S}=1$, which comprise the sequences A and B1 (cf. Table 3), diffusive mixing dominates the advective mass transport in the inner zones of the disk ( $r \lesssim 4 \mathrm{AU})$. Further outside ( $r \gtrsim 4 \mathrm{AU})$, the dominant process for mass transport is advection. At the radial distance, $r=4 \mathrm{AU}$, the advective and the diffusive timescales coincide and are equal to roughly $1300 \mathrm{yr}$. The implications of this important fact on the distribution of the various chemical species are discussed in more detail below.

Equation (47) shows that there is a relatively strong (quadratic) dependence of $r_{\text {mix }}$ on the ratio, $\beta / u_{r}$. However, the strength of the large-scale circulation is expected to increase with increasing $\beta$, that is, the higher (lower) the parameter $\beta$ is chosen, the faster (slower) the motions in the circulation will be. It could even be that $\beta / u_{r}$ is essentially constant for $\beta$ varying in between $10^{-4}$ and $10^{-2}$. For the moment being, of course, taking this possibility into consideration is nothing more than a wild guess. Further extended numerical experiments are necessary to clarify this interesting issue.

Mixing processes in the vertical direction are of equal importance. Due to the well-ordered large-scale circulation the $\mathrm{ad}$ vective exchange of mass elements belonging to the outgoing equatorial stream with those of the incoming high-altitude flow will be rather ineffective. Mixing in the vertical direction is performed almost entirely by diffusion. We now can estimate the respective diffusion timescale, $t_{\mathrm{diff}}^{\perp}$, by re-interpreting the rather unspecified length scale $\Delta r$ in (46) to be the typical vertical length scale of the disk, for which twice the disk's half-thickness, $h:=c_{\mathrm{s}} / \Omega$, may serve as an appropriate estimate. This yields

$t_{\mathrm{diff}}^{\perp}=\frac{2 \Pi}{\pi \beta} \cdot \mathcal{S} \cdot\left(\frac{h}{r}\right)^{2}$.

For our numerical simulations with the given standard parameters, $\beta=10^{-3}, \mathcal{S}=1$, together with the calculated relative half-thickness, $h / r \simeq 0.10 \ldots 0.12$ (cf. Fig. $7 b$ ), we have $t_{\text {diff }}^{\perp} / \Pi=5 \ldots 9$, which leads to diffusive mixing timescales roughly in between $5 \mathrm{yr}$ (at $1 \mathrm{AU}$ ) and $100 \mathrm{yr}$ (at $5 \mathrm{AU}$ ) across our model disk in the radial direction. So we can safely state that at the reference epoch of $300 \mathrm{yr}$ the distribution of the various chemical species in the vertical direction is, to a large extent, modulated by diffusive mixing.

\subsubsection{Relation to 1-D models}

As a link to the one-zone and (1+1)-D approximation, Fig. 7 displays the radial stratification of some physical quantities integrated over the vertical $(z-)$ coordinate and other quantities related to the vertically outgoing radiative flux, $F_{z}$. In particular, panels (a)-(c) show the disk's surface density,

$\Sigma \equiv \Sigma(r):=2 \int_{0}^{z_{\max }} \rho(r, z) \mathrm{d} z$

its relative "half-thickness",

$\frac{h}{r}:=\frac{1}{2 r} \cdot \frac{\Sigma(r)}{\rho(r, z=0)}$,

and the net accretion rate,

$\dot{M} \equiv \frac{\mathrm{d} M}{\mathrm{~d} t}:=4 \pi r \int_{0}^{z_{\max }} \rho(r, z) u_{r}(r, z) \mathrm{d} z$,

respectively. Figure $7 d$ displays two quantities that are related to the effective temperature, $T_{\text {eff }}$. The full line represents the radial dependence of the gas temperature, $T\left(r, \tau_{z}\right)$, at the optical depth, $\tau_{z}:=\tau_{z}(r)=1$ (cf. Fig. 5, third left panel), counted from the "upper" boundary at $z=z_{\max } \equiv 1.5 \mathrm{AU}$ "down" to the equatorial plane at $z=0$ in the vertical direction. The quantity, $T\left(r, \tau_{z}\right)$, ought to be close to $T_{\text {eff }}$ which is, by definition, the equivalent black-body temperature corresponding to the outgoing radiative flux, $F_{z}\left(r, z=z_{\max }\right)$, in the vertical direction, thus

$\sigma T_{\mathrm{eff}}^{4}=F_{z}\left(r, z=z_{\max }\right)$

The dashed line depicts the radial variation of the effective temperature, $T_{\text {eff }}$, as defined in Eq. (52) above. For $r \gtrsim 1.8 \mathrm{AU}$ the coincidence between the two curves is, indeed, almost perfect. A major deviation occurs only at lower radial distances.

\subsubsection{Numerical constraints and demands}

The main reason for this behavior of the numerical solution for $r \lesssim 1.8 \mathrm{AU}$, which is also indicated by the sudden, relatively steep decrease of the disk's relative half-thickness, $h / r$ (cf. Fig. 7b), toward the inner boundary (situated at $r_{0}=$ $0.8 \mathrm{AU})$, is the extremely poor numerical resolution of the pressure-supported hydrostatic quasi-equilibrium in the vertical direction. Only 7-10 gridpoints are available to cover 5-6 orders of magnitude in the density or gas pressure. Adjacent numerical cells exhibit large changes in the optical depth, there are big jumps from heavily opaque to fairly transparent layers where the Eddington approximation must be most probably modified by using an appropriate, but otherwise rather artificial, flux limiter. In this way, large numerical errors are introduced. This can be avoided only if, first of all, the numerical resolution is decisively improved and, equally important, the flux-limited Eddington approximation is replaced by a more sophisticated treatment of radiative transfer.

Despite this somewhat uncomfortable, but natural, lack of numerical resolution and accuracy in the vicinity of the inner boundary, we can fairly state that the code has proved to be extremely stable and robust. In the simulations carried out so far an exceptional stop never occurred, neither was any "fine-tuning" of control parameters ever necessary to continue the calculation. We presume that the main stabilizing effect in our numerical operator splitting scheme relates to the implicit source step for solving the energy balance equations. There, the (kinetic) temperature, pressure and internal energy of the gas, and the radiation energy density are iteratively determined - under the constraint of constant (known) mass density and velocity field - so as to yield a set of intrinsically consistent physical quantities.

In other words, on the source-step level the gas pressure is controlled by taking the total energy balance into account, which would not be possible within the framework of an explicit scheme. On the other hand, it is worth noting that, according to the so-called pressure-correction method, it is known that for far subsonic, i.e., nearly incompressible, flows - which are typical in quasi-stationary accretion disks - the gas pressure must be determined as the solution of a certain Helmholtz-type elliptic differential equation (see, e.g., Harlow \& Amsden 1968; Bijl \& Wesseling 1998; Keller 2003) in order to warrant numerical stability. Again, besides fulfilling the equation of state, which is the trivial part of the game, an implicit step for solving the associated Dirichlet boundary-value problem is necessary. To which extent there might (or might not) exist a logical connection between this pressure-correction method and the implicit one we have been using in our calculations is an exciting mathematical question. 
In physical terms, the intermediate implicit source step which is, after all, the most costly part of the update scheme for one timestep, causes an effective damping, or even complete suppression, of the spurious small-scale pressure waves that are continually generated by unavoidable discretisation errors. Since the advection terms are negligibly small in extremely subsonic regimes, no efficient damping mechanism is inherent to explicit schemes. This is why purely explicit methods applied to lowMach flows tend to steepen these waves, particularly in stratified media. As a most undesirable result, an artificial small-scale velocity field would build up giving rise to an unstable numerical solution which becomes wiggly or even saw-toothed already after a few timesteps and does not at all converge to the, in any case, smooth physical solution.

During the violent dynamical switch-on phase the (nonlinear) advection terms are important. This is why they deserve a careful numerical treatment. The operator-splitting method of Norman \& Winkler (1986) we have adapted for our purposes makes use of the spatially second-order monotonic van Leer $(1977,1979)$ advection scheme which has proven to be decisively superior to the old-fashioned first-order donor-cell approximation as is discussed in detail by LeVeque et al. (1998). We can thus be sure that the advection errors remain small. Hence, our treatment of the advection terms would neither bias the numerical results referring to the dynamical phase nor, all the less, the most important findings pertaining to the quiescent, viscosity-dominated long-term evolution of the disk.

\subsubsection{Chemistry and mixing}

As has already been pointed out, the main goal of carrying out calculations for the model sequences A, B1 and B2 (see Table 3) was twofold: firstly, to test the relation between advective and diffusive mass transport by varying the Schmidt number, $\mathcal{S}$, and, secondly, to identify and investigate those chemical reactions which could play an important role for producing a sufficient amount of carbon dioxide $\left(\mathrm{CO}_{2}\right)$ in preplanetary nebulae. It is worth noting again that the primordial material with which we start out only contains molecular hydrogen $\left(\mathrm{H}_{2}\right)$ as the dominant gaseous component with an admixture of carbon monoxide (CO) and water $\left(\mathrm{H}_{2} \mathrm{O}\right)$ frozen out on the dust grains (cf. Table 2). Any other species participating actively in the C-, H-, O-chemistry, among them $\mathrm{CO}_{2}$, appear later in the evolution of the disk.

Let us first discuss the "realistic" case A. In the same manner as arranged in Fig. 4, the 10 individual panels of Fig. 8 display the distribution of the logarithmic abundance (per weight), $\log c_{X}$, of each reactive gaseous species, $X$, over the $(r, z)$-plane. Because of the very low abundance of $\mathrm{C}$ (gaseous), $\mathrm{HO}_{2}$, and $\mathrm{H}_{2} \mathrm{O}_{2}\left(\lesssim 10^{-15}\right)$, these species are not plotted. As can be read off from the (log-)density contours in Figs. 5 and 6 the disk proper is confined to the region, $|z| / r \lesssim 0.25 \ldots 0.3$; there is only a tiny amount of "frozen" material kept "above" the disk's surface for technical reasons ("vacuum" density, $\rho_{\mathrm{vac}}$, cf. Sect. 4.4), which is physically irrelevant. In this quasi-vacuum region, only the primordial concentrations of the gaseous species, $\mathrm{H}_{2}, \mathrm{H}_{2} \mathrm{O}$, and $\mathrm{CO}$ are retained.

The depletion of water vapor onto dust grains at temperatures below about $150 \mathrm{~K}$, indicated by the steep decrease of gaseous $\mathrm{H}_{2} \mathrm{O}$, is visible in the right upper corner of the respective panel with label "H2O" in Fig. 8. This structure exhibiting enhanced opacity (cf. Fig. 9) related to ice-coated dust grains is limited by the "snow line" (cf. Fig. 5) and is vaguely alike a terrestrial high-altitude ice cloud. By contrast, in the hot $\left(T \gtrsim 10^{3} \mathrm{~K}\right)$ innermost regions at radial distances smaller than about 2 AU and a vertical extension of about one scale-height $(|z| / r \lesssim 0.15)$ atomic hydrogen $(\mathrm{H})$ and the hydroxyl radical $(\mathrm{OH})$ have already become moderately abundant. A good deal less prominent is the abundance of atomic oxygen $(\mathrm{O})$. Because of its low abundance relative to $\mathrm{OH}$, it is expected that the combustion of the microscopic soot/graphite particles will be dominated by surface reactions with $\mathrm{OH}$ to form the ketenyl radical $\left(\mathrm{HC}_{2} \mathrm{O}\right)$. The ensuing gas-phase chemistry would then yield a large diversity of hydrocarbons. All other species $-\mathrm{O}_{2}, \mathrm{HO}_{2}$, $\mathrm{H}_{2} \mathrm{O}_{2} ; \mathrm{H}_{2} \mathrm{CO}, \mathrm{HCO}$ - remain so much less abundant in the disk proper and are only important as intermediate products of the chemical reaction network.

In Fig. 11, we summarize the results of the two comparative model calculations labelled B1 and B2, in order to find out to which extent diffusive transport of material is an important mixing mechanism. As estimated by Eq. (48), diffusive mixing in the vertical direction is expected to be the dominant transport process. Already a glimpse to Fig. 11-B2 (diffusive transport switched off; Schmidt number $\mathcal{S}=\infty$ ) and Fig. 11-B1 ("realistic" transport; $S=1$ ) is sufficient to prove the validity of this assertion. The advective transport in the radial direction is nicely visible as the distortion of the contour lines above one half-thickness of the disk, where the inflow velocities are relatively high.

Comparing the Figs. 11 and 8 (cf. the respective panels for $\mathrm{CO}_{2}$ ) reveals immediately a possible significance of the water-gas shift reaction for a large-scale distribution of $\mathrm{CO}_{2}$ in preplanetary nebulae - provided it becomes ever efficient, which could happen only if an appropriate catalytic agent, like microscopic dust grains consisting of elementary iron, were available. If the water-gas shift reaction is turned down to realistic rates (model sequence $\mathrm{A}$ ) the remaining $\mathrm{C}-, \mathrm{H}-$, O-chemistry will yield $\mathrm{CO}_{2}$ predominantly in the hot inner regions (cf. Fig. 8).

\section{Final remarks and outlook}

In this paper we have documented first steps toward constructing fully 2-D models of preplanetary disks including chemical reactions and diffusive mixing of the various chemical species. The results of the numerical experiments verify the remarkable robustness and numerical stability of the hydrodynamical code. It is based on a combination of an explicit operator-splitting method, that is, by taking consecutively the source step and the transport step into account, with an implicit solver of the energy balance equation including the gas pressure. The solution of the chemical network is obtained at the source-step level. Thus, for each numerical $(r, z)$-cell a system of stiff ordinary differential equations for the abundance of the various chemical species (see Eq. (23)) is solved independently. It is obvious that this particular feature of the numerical method allows for a massive parallelization of the code. However, the main advantage of the numerical procedure is its uniform efficiency in dealing with either high or low Mach number flow types.

The following results are the main outcome of the numerical experiments:

1. The formation of a stable large-scale circulation within the disk, i.e., a slow "equatorial" flow and a faster "highaltitude" stream in the outward and inward direction, respectively, superimposing the net accretion flow (cf. Fig. 7).

2. The importance of advective transport of material in the radial direction as compared to diffusive mixing at larger radial distances, i.e., the existence of a finite radial distance, $r_{\text {mix }}$, beyond which radial advective transport dominates diffusive 
B1
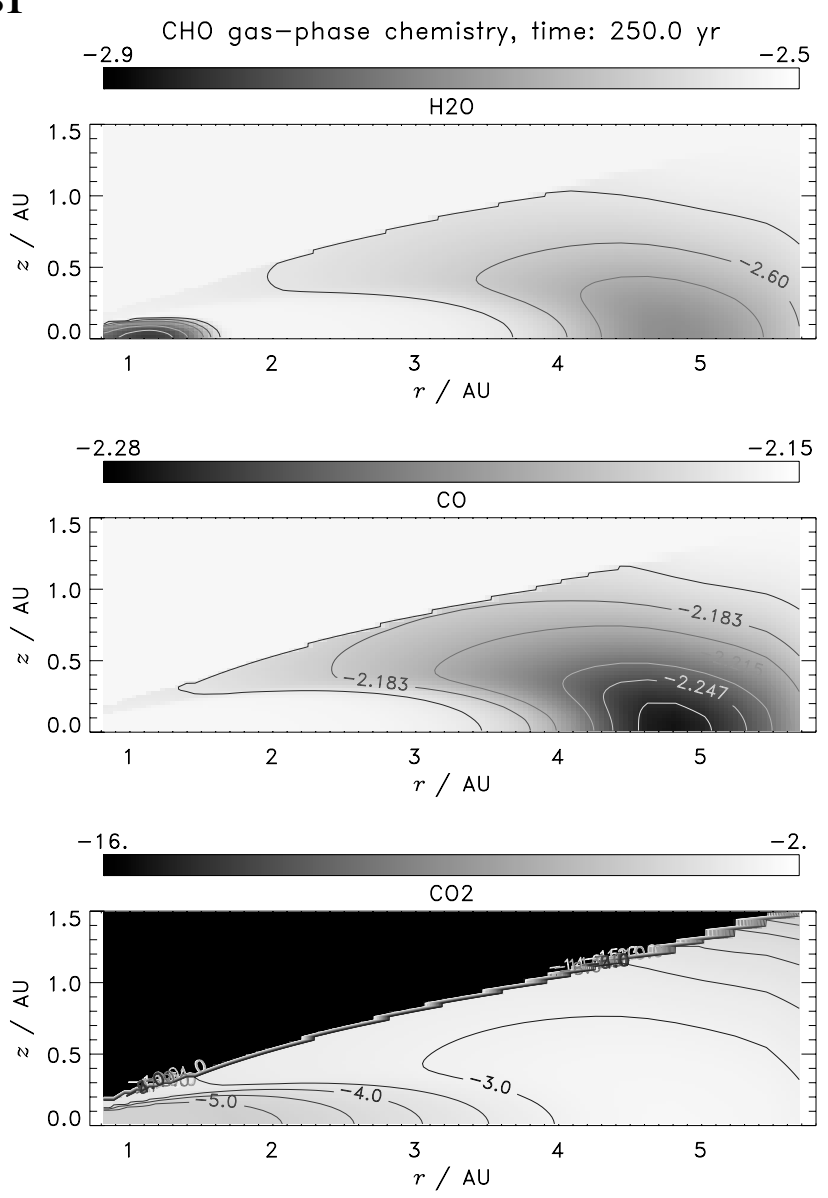

B2
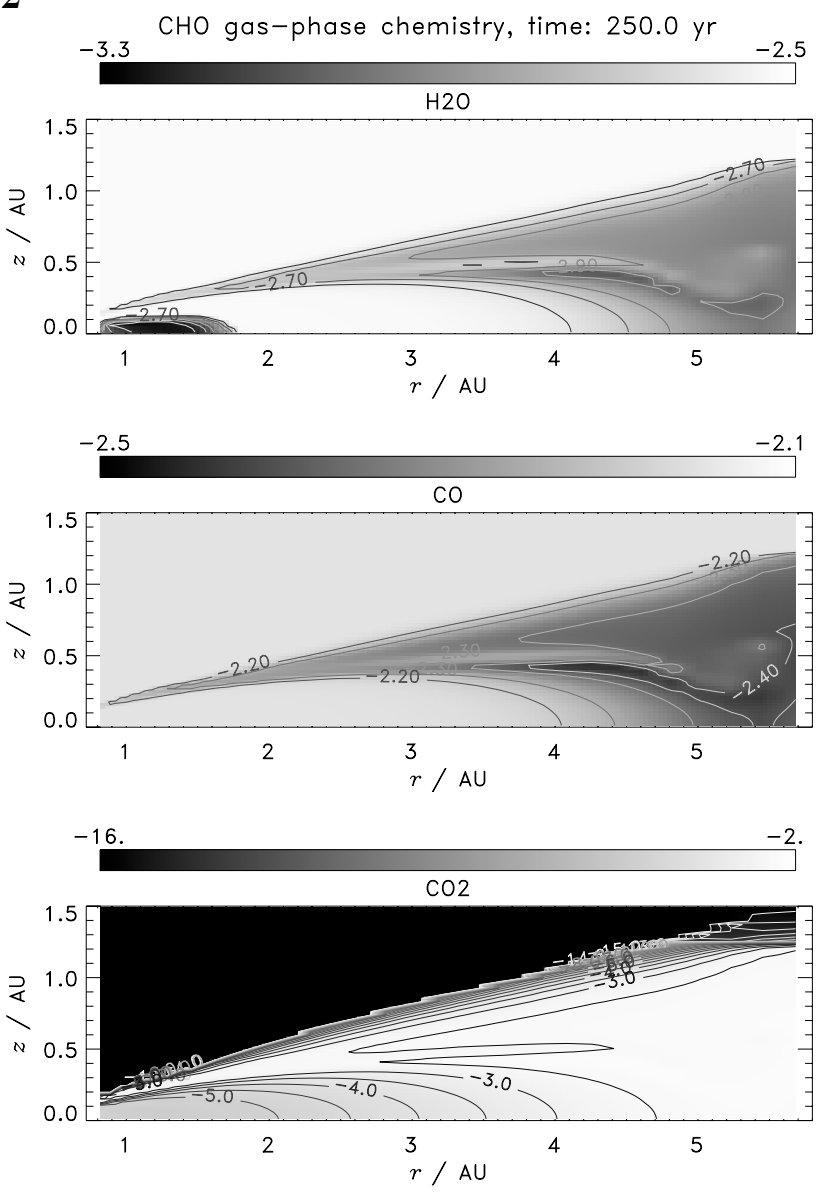

Fig. 11. Distribution of the (except $\mathrm{H}_{2}$ ) three most abundant species $\mathrm{CO}, \mathrm{CO}_{2}, \mathrm{H}_{2} \mathrm{O}$ for model category $\mathrm{B}$ - with $\mathrm{B} 1$ and $\mathrm{B} 2$ referring to the $\mathrm{Schmidt}$ constant, $\mathcal{S}=1$ and $\mathcal{S}=\infty$ (diffusive mixing switched off), respectively (cf. Table 3 ) - after $250 \mathrm{yr}$, illustrating that diffusion is the dominating mixing process. Note that the total concentration (vapor + ice) of the water molecule $\left(\mathrm{H}_{2} \mathrm{O}\right)$ is shown.

mixing (see Eq. (47)), provided a stable long-lasting largescale circulation is maintained.

3. The general importance of diffusive mixing in the vertical direction (see Eq. (48)).

4. The successful coupling of evaporation and condensation processes referring to both refractory grains and their volatile (water-)ice mantles with the opacity, at least in the simplified approximation of instantaneously established equilibrium according to the local vapor pressure of silicates (the vapor pressure of iron is taken as a useful substitute) and water, respectively.

Simulations relating to more massive preplanetary disks containing up to $0.39 M_{\odot}$, as has recently been derived from $3 \mathrm{~mm}-$ observations of disks around several stellar objects belonging to the Orion Nebula cluster (Eisner \& Carpenter 2006), demand the implementation of an appropriate Poisson solver in the 2-D code. This will be the first task to be accomplished for improving on the hydrodynamical component of the code.

Concerning the last item above, the extension of our simplified evaporation and condensation models to more general non-equilibrium models is almost straightforward. The only big uncertainties refer to the lack of reliable data for the heteromolecular gas-solid chemistry with its various reaction paths on the surface of silicate grains. Somewhat more comfortable is the situation for homomolecular grain growth at low temperatures, i.e., the formation and destruction of ice mantles consisting of frozen water, carbon monoxide, and further volatile species.

Another important solid-gas reaction process to be implemented in the next model series is the combustion of the submicroscopic graphite/soot particles which form the most conspicuous dust component following the silicate grains. Carbon combustion is the only way to form the hydrocarbons that are found in cometary material. At temperatures above 800-900 K, that is, in the innermost parts of the disk, the surface reaction with the hydroxyl radical $(\mathrm{OH})$ yields the ketenyl radical $\left(\mathrm{HC}_{2} \mathrm{O}\right)$ which, in turn, serves as the starting compound for building up a large number of hydrocarbons (e.g., Finocchi et al. 1997; Gail 2002). Subsequent outward transport of the material into the outer, cold regions of the preplanetary disk make the hydrocarbons, together with other volatile species like $\mathrm{H}_{2} \mathrm{O}$, $\mathrm{CO}$, etc., condense out on the refractory grains. In summary, all these processes give rise to the specific composition of the raw material, out of which cometary bodies eventually form.

A further challenge is to take coagulation of the dust grains into account. Changing the particle size distribution and forming dust agglomerates of different composition have great influence on the optical properties and, hence, on the opacity of the material. Mineralogical and physicochemical processes, e.g., annealing and diffusion or chemical transition fronts in dust grains, respectively, will change their absorption properties considerably, too. Sooner or later, all these processes must be taken into consideration in the calculations so as to have a chance to arrive at 
realistic models of preplanetary nebulae whose properties can be confronted with the empirical findings.

For the time being, it is not feasible to cover the evolution of preplanetary disks in their whole spatial extension (in between $0.01 \mathrm{AU}$ and $100 \mathrm{AU}$ ) and time span of the order of $10^{6} \mathrm{yr}$ with the existing code. The limiting factor is the restrictive Courant-Friedrichs-Lewy (CFL-)condition on the timestep for explicit numerical schemes. Fully implicit 2-D codes, which would not be subject to the CFL-condition, are so much less available if additional source terms like chemical reactions are to be taken into account. Domain decomposition with additional inner boundary or fit conditions will probably improve the situation to some extent without claiming to aim, in this way, at a universal solution of the overall problem. Nevertheless, elaborate methods to investigate $2-\mathrm{D}$ models may serve, on the one hand, as a useful supplement to (1+1)-D studies, and, on the other hand, as a firm guideline for the construction of conclusive 3 -D preplanetary disk models in the future.

Acknowledgements. We thank our colleague W. J. Duschl for useful hints to improve the readability of the text and for enlightening discussions on the true meaning of the $\beta$-prescription for the turbulent viscosity. We are also grateful to the (anonymous) referees for constructive suggestions and remarks. This work was sponsored by the Heidelberg Collaborative Research Centres SFB 359 "Reactive Flows, Diffusion and Transport" and SFB 439 "Galaxies in the Young Universe", which have been supported by the Deutsche Forschungsgemeinschaft (DFG).

\section{References}

Alexander, D. R., \& Ferguson, J. W. 1994, ApJ, 437, 879

Azatyan, V. V., Aleksandrov, E. N., \& Troshin, A. F. 1975, Kinet. Catal., 16, 306 Baulch, D. L., Cobos, C. J., Cox, R. A., et al. 1992, J. Phys. Chem. Ref. Data, 21,411

Bell, K. R., \& Lin, D. N. C. 1994, ApJ, 427, 987

Bell, K. R., Cassen, P. M., Klahr, H. H., \& Henning, T. 1997, ApJ, 486, 372

Bijl, H., \& Wesseling, P. 1998, J. Comp. Phys., 141, 153

Cassen, P. M. 1994, Icarus, 112, 405

Castor, J. I. 1986, in NATO ASI Series C. Mathematical and Physical Sciences, Vol. 188, Astrophysical Radiation Hydrodynamics, ed. K.-H. Winkler, \& M. L. Norman (Dordrecht: Reidel Publishing Company), 1

Chase Jr., M. W., ed. 1998, NIST-JANAF thermochemical tables, 4th edn., J. Phys. Chem. Ref. Data, monograph No. 9 (National Institute of Standards and Technology, US)

Cohen, N., \& Westberg, K. R. 1983, J. Phys. Chem. Ref. Data, 12, 531

Dalgarno, A., Du, M. L., \& You, J. H. 1990, ApJ, 349, 675

Duschl, W. J., Strittmatter, P. A., \& Biermann, P. L. 2000, A\&A, 357, 1123

Eisner, J. A., \& Carpenter, J. M. 2006, ApJ, 641, 1162

Eiteneer, B., Yu, C.-L., Goldenberg, M., \& Frenklach, M. 1998, J. Phys. Chem. A, 102,5196

El-Gamal, M. 1995, Ph.D. Thesis, University of Stuttgart

Fairbairn, A. R. 1969, Proc. R. Soc. London A, 312, 207

Fegley Jr., B., \& Prinn, R. G. 1989, in The Formation and Evolution of Planetary Systems, ed. H. A. Weaver, \& L. Danley (Cambridge: Cambridge University Press), 171
Ferguson, J. W., Alexander, D. R., Allard, F., et al. 2005, ApJ, 623, 585 Finocchi, F., \& Gail, H.-P. 1997, A\&A, 327, 825

Finocchi, F., Gail, H.-P., \& Duschl, W. J. 1997, A\&A, 325, 1264

Ford, P. C. 1981, Acc. Chem. Res., 14, 31

Frank, J., King, A., \& Raine, D. 2002, Accretion Power in Astrophysics, 3rd edn. (Cambridge University Press)

Gail, H.-P. 1998, A\&A, 332, 1099

Gail, H.-P. 2001, A\&A, 378, 192

Gail, H.-P. 2002, A\&A, 390, 253

Gail, H.-P. 2003, Lecture Notes in Physics (Berlin: Springer Verlag), 609, 55

Geballe, T. R., Knapp, G. R., Leggett, S. K., et al. 2002, ApJ, 564, 466

Günther, R. 1974, Verbrennung und Feuerungen (Berlin, Heidelberg, New York: Springer-Verlag)

Harlow, F. H., \& Amsden, A. A. 1968, J. Comp. Phys., 3, 80

Keeley, D. A. 1970, ApJ, 161, 643

Keller, C. 2003, Ph.D. Thesis, University of Heidelberg

Keller, C., \& Gail, H.-P. 2004, A\&A, 415, 1177

Kiefer, J. H. 1974, J. Chem. Phys., 61, 244

Kippenhahn, R., \& Thomas, H.-C. 1982, A\&A, 114, 77

Kley, W., Papaloizou, J. C. B., \& Lin, D. N. C. 1993, ApJ, 416, 679

Kraus, J. 2003, Ph.D. Thesis, Institut für Siedlungswasserwirtschaft, University of Karlsruhe

Lahuis, F., van Dishoeck, E. F., Boogert, A. C. A., et al. 2006, ApJ, 636, L145

Le Teuff, Y. H., Millar, T. J., \& Markwick, A. J. 2000, A\&AS, 146, 157

LeVeque, R. J., Mihalas, D., Dorfi, E. A., \& Müller, E. 1998, Computational Methods for Astrophysical Fluid Flow, Saas-Fee Advanced Course 27, Lecture Notes 1997, Swiss Society for Astrophysics and Astronomy (Springer Verlag)

Lichtenegger, H. I. M., \& Kömle, N. I. 1991, Icarus, 90, 319

Lifschitz, A., \& Michael, J. V. 1991, Symp. Int. Combust. Proc., 23, 59

Lloyd, A. C. 1974, Int. J. Chem. Kinet., 6, 169

Marigo, P. 2002, A\&A, 387, 507

Mathis, J. S., Rumpl, W., \& Nordsieck, K. H. 1977, ApJ, 217, 425

Mayer, S. W., \& Schieler, L. 1968, J. Phys. Chem., 72, 2628

Mitchell, G. F. 1984, ApJS, 54, 81

Norman, M. L., \& Winkler, K.-H. 1986, in NATO ASI Series C. Mathematical and Physical Sciences, Vol. 188, Astrophysical Radiation Hydrodynamics, ed. K.-H. Winkler, \& M. L. Norman (Dordrecht: Reidel Publishing Company), 187

Patzer, A. B. C. 2004, in Astronomical. Soc. Pacific Conf. Ser., Vol. 309, Astrophysics of Dust, ed. A. N. Witt, G. C. Clayton, \& B. T. Draine (San Francisco: ASP), 301

Regev, O., \& Gitelman, L. 2002, A\&A, 396, 623

Różyczka, M., Bodenheimer, P., \& Bell, K. R. 1994, ApJ, 423, 736

Ruden, S. P., \& Lin, D. N. C. 1986, ApJ, 308, 883

Ruden, S. P., \& Pollack, J. B. 1991, ApJ, 375, 740

Schmitt, W., Henning, T., \& Mucha, R. 1997, A\&A, 325, 569

Shakura, N. I., \& Sunyaev, R. A. 1973, A\&A, 24, 337

Sharp, C. M., \& Huebner, W. M. 1990, ApJS, 72, 417

Siemiginowska, A. 1988, Acta Astron., 38, 21

Talbi, D., \& Herbst, E. 2002, A\&A, 386, 1139

Tsang, W., \& Hampson, R. F. 1986, J. Phys. Chem. Ref. Data, 15, 1087

Turner, N. J., Willacy, K., Bryden, G., \& Yorke, H. W. 2006, ApJ, 639, 1218

Urpin, V. A. 1984, Soviet Ast., 28, 50

van Leer, B. 1977, J. Comput. Phys., 23, 276

van Leer, B. 1979, J. Comput. Phys., 32, 101

Voshchinnikov, N. V., Il'in, V. B., Henning, T., \& Dubkova, D. N. 2005, A\&A, 429,371

Voshchinnikov, N. V., Il'in, V. B., Henning, T., \& Dubkova, D. N. 2006, A\&A, 445,167 